Florida International University FIU Digital Commons

FIU Electronic Theses and Dissertations

University Graduate School

6-28-2016

\title{
Evaluation of Surface Layer Parameterizations Using In-Situ Observations
}

Jeremy Katz

Florida International University, jkatz029@fiu.edu

DOI: $10.25148 /$ etd.FIDC000720

Follow this and additional works at: https:// digitalcommons.fiu.edu/etd

Part of the Atmospheric Sciences Commons, and the Meteorology Commons

\section{Recommended Citation}

Katz, Jeremy, "Evaluation of Surface Layer Parameterizations Using In-Situ Observations" (2016). FIU Electronic Theses and Dissertations. 2604.

https://digitalcommons.fiu.edu/etd/2604

This work is brought to you for free and open access by the University Graduate School at FIU Digital Commons. It has been accepted for inclusion in FIU Electronic Theses and Dissertations by an authorized administrator of FIU Digital Commons. For more information, please contact dcc@fiu.edu. 


\title{
FLORIDA INTERNATIONAL UNIVERSITY
}

Miami, Florida

\section{EVALUATION OF SURFACE LAYER PARAMETERIZATIONS USING IN-SITU OBSERVATIONS}

\author{
A thesis submitted in partial fulfillment of \\ the requirements for degree of \\ MASTER OF SCIENCE \\ in \\ GEOSCIENCES \\ by \\ Jeremy Katz
}

2016 
To: Dean Michael R. Heithaus

College of Arts, Sciences and Education

This thesis, written by Jeremy Katz, and entitled Evaluation of Surface Layer Parameterizations Using In-Situ Observations, having been approved in respect to style and intellectual content, is referred to you for judgment.

We have read this thesis and recommend that it be approved.

$\begin{array}{r}\hline \text { Haiyan Jiang } \\ \hline \text { Hugh Willoughby } \\ \hline \text { Ping Zhu, Major Professor }\end{array}$

Date of Defense: June 28, 2016

The thesis of Jeremy Katz is approved.

Dean Michael R. Heithaus College of Arts, Sciences and Education

Andrés G. Gil

Vice President for Research and Economic Development and Dean of the University Graduate School

Florida International University, 2016 


\section{ACKNOWLEDGMENTS}

I wish to thank the members of my committer for their support, patience, and guidance that they provided me. I am grateful for how they challenged me to continually improve myself and improve the quality of my work as well. I would like to thank my major professor, Dr. Ping Zhu. For always showing his support and his belief in my abilities as a student. I also want to thank my family, who gave me love and support me, despite being so far away from home for long periods of time. I want to thank my friends for their support and understanding of when I would have to cancel plans with them to work. 


\section{ABSTRACT OF THESIS}

\section{EVALUATION OF SURFACE LAYER PARAMETERIZATIONS USING IN-SITU OBSERVATIONS}

by

Jeremy Katz

Florida International University, 2016

Miami, Florida

\section{Professor Ping Zhu, Major Professor}

Appropriate calculation of surface turbulent fluxes between the atmosphere and the underlying surface is one of the major challenges in geosciences. In practice, the surface turbulent fluxes are estimated from the mean surface meteorological variables based on the bulk transfer model combined with Monnin-Obukhov Similarity (MOS) theory. Few studies have been done to examine the extent that to which this flux parameterization framework can be applied to different weather and surface conditions. A novel validation method is developed in this thesis research, which is applied to evaluate the surface flux parameterization using in-situ observations. The main findings are: (a) the theoretical prediction that uses MOS theory does not match well with those directly computed from the observations collected in the coastal region. (b) Large spread in exchange coefficients mainly occurs in the calm wind regime with strong stability. (c) Large turbulent eddies, which depend largely on the mean flow and surface conditions, tend to break the constant flux assumption in the surface layer. 


\section{TABLE OF CONTENTS}

CHAPTER

PAGE

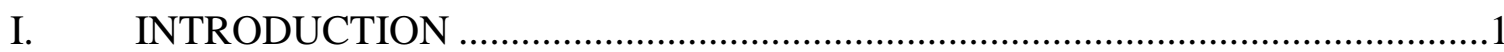

1.1 Turbulence and the Atmospheric Boundary Layer .................................1

1.2 The Bulk Transfer Parameterization of Surface Turbulent Fluxes ..........3

II. METHODOLOGY IN EVALUATION OF SURFACE FLUX

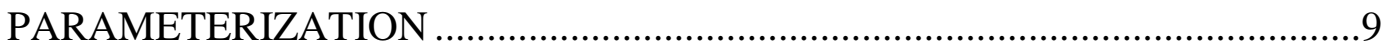

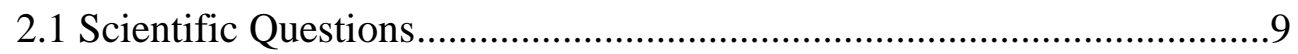

2.2 A new framework for evaluating surface flux parameterization.............11

III. INSTRUMENTATION, OBSERVATION, AND DATA PROCESSING ............17

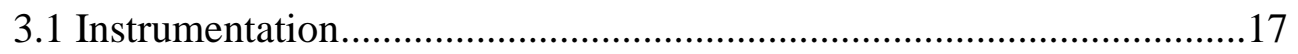

3.2 Tower Deployment in SCOPE Field Experiment .................................20

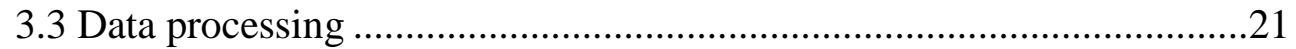

3.3.1 Eddy Correlation Method .....................................................21

3.3.2 Quality Control and Assurance Procedures .........................22

a. $\quad$ Spike Removal.......................................................22

b. Sonic Anemometer Tilting Correction ......................23

c. Consistency Checks..................................................26

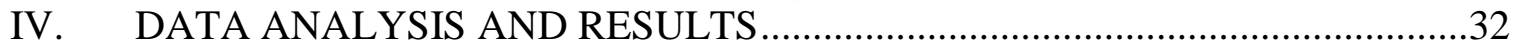

4.1 Overview of SCOPE Tower Observations ............................................32

4.2 Evaluation of Surface Flux Parameterization ........................................34

I.3 Issues Regarding Basic Assumptions of MOS ....................................37

I.3.1 Von-Karman Constant...........................................................37

I.3.2 Constant Flux Assumption in the SL ..................................42

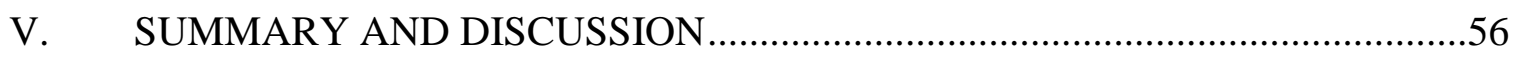

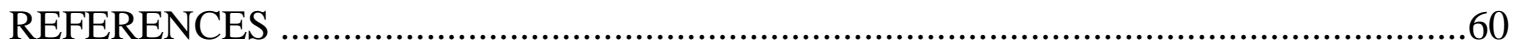

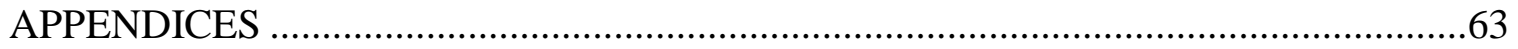




\section{LIST OF FIGURES}

1.1 Schematic illustration of the vertical heat fluxes induced by turbulent eddies........8

2.1 Drag coefficients in near neutral conditions ............................................ 15

2.2 Time-averaged turbulent motion over a rough terrain .....................................16

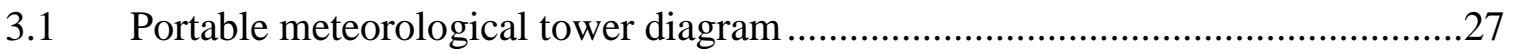

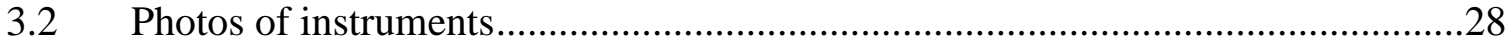

3.3 Aerial view of PWT deployment at Beasley Park, Fort Walton Beach FL ..........29

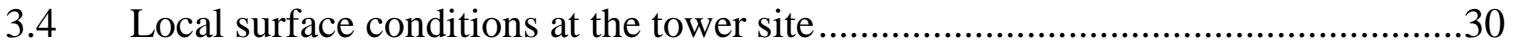

3.5 Error caused by misalignment of sonic anemometer (after Mahrt 2010) .............31

4.1 Time series of 15 -min mean of observations ..............................................45

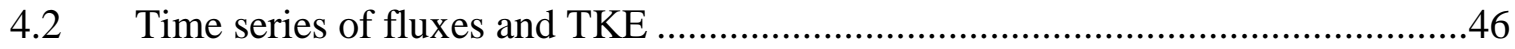

4.3 Comparison of fluxes and TKE at two heights .........................................47

4.4 Observational and theoretical adjusted drag coefficients .................................48

4.5 Observational and theoretical adjusted exchange coefficient for heat.................49

4.6 Observational and theoretical adjusted exchange coefficient for moisture ...........50

4.7 Observational data sorted based on wind speed observations ............................51

4.8 TKE as a function of $u_{*}^{2}$ from different field experiments including SCOPE ......52

4.9 Power spectra of $u, v$, and $w$ for an arbitrary 15-minute spectral lag ..................53

4.10 Estimated coefficient $\eta=c_{1}^{3 / 2} c_{2}$ against wind speed.....................................54

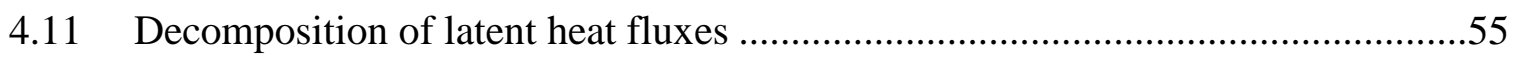




\section{ABBREVIATIONS AND ACRONYMS}

\begin{tabular}{|c|c|}
\hline $\mathrm{ABL}$ & Atmospheric Boundary Layer \\
\hline$C_{D}$ & Bulk transfer coefficient for momentum, drag coefficient (dimensionless) \\
\hline$C_{D a}$ & Adjusted dimensionless exchange coefficient for momentum \\
\hline$C_{D N}$ & Neutral drag coefficient \\
\hline$C_{H}$ & Bulk transfer coefficient for heat (dimensionless) \\
\hline$C_{H a}$ & Adjusted dimensionless exchange coefficient for heat \\
\hline$C_{Q}$ & Bulk transfer coefficient for moisture (dimensionless) \\
\hline$C_{Q a}$ & Adjusted dimensionless exchange coefficient for moisture \\
\hline DR & Double rotation method \\
\hline FCMP & Florida Coastal Monitoring Program \\
\hline FFT & Fast Fourier Transform \\
\hline FNL & Final \\
\hline GoMRI & Gulf of Mexico Research Initiative \\
\hline HWRF & Hurricane Weather Research and Forecasting \\
\hline IHRC & International Hurricane Research Center (FIU) \\
\hline$L$ & Obukhov Length (m) \\
\hline LHS & Left hand side \\
\hline MOS & Monin Obukhov Similarity (theory) \\
\hline$P$ & Pressure $(\mathrm{hPa})$ \\
\hline PWT & Portable Wind Tower \\
\hline$q$ & Water Vapor Mixing Ratio \\
\hline
\end{tabular}




\begin{tabular}{|c|c|}
\hline$q_{G}$ & "Skin" Water Vapor Mixing Ratio \\
\hline RHS & Right hand side \\
\hline SCOPE & Surfzone Coastal Oil Pathways Experiment \\
\hline SL & Surface Layer \\
\hline SLPM & Standard liters per minute \\
\hline$T$ & Temperature $\left({ }^{\circ} \mathrm{C}\right)$ \\
\hline TKE & Turbulent Kinetic Energy \\
\hline$T_{s}$ & Sonic temperature $\left({ }^{\circ} \mathrm{C}\right)$ \\
\hline$T_{v}$ & Virtual temperature $\left({ }^{\circ} \mathrm{C}\right)$ \\
\hline$t$ & Time (unit varies) \\
\hline$U$ & Total wind $\left(\mathrm{ms}^{-1}\right)$ \\
\hline$u_{*}$ & Friction velocity $\left(\mathrm{ms}^{-1}\right)$ \\
\hline$u$ & Along wind component $\left(\mathrm{ms}^{-1}\right)$ \\
\hline$v$ & Cross wind component $\left(\mathrm{ms}^{-1}\right)$ \\
\hline$w$ & Vertical wind component $\left(\mathrm{ms}^{-1}\right)$ \\
\hline WMP & WindMaster Pro (Sonic Anemometer) \\
\hline WRF & Weather Research and Forecasting \\
\hline$z$ & Height above ground measurements are observed $(10 \mathrm{~m})$ \\
\hline$z_{0}$ & Aerodynamic roughness length (m) \\
\hline$\theta$ & Potential Temperature \\
\hline$\theta_{G}$ & "Skin" Potential Temperature \\
\hline$\theta_{v}$ & Virtual Potential Temperature \\
\hline
\end{tabular}


$\zeta$

$\kappa$

$\varepsilon_{\mathrm{e}}$

$\Lambda$

Surface layer stability parameter (dimensionless)

Von-Karman constant

TKE dissipation rate

Empirical dissipation length scale 


\section{Chapter I: INTRODUCTION}

\subsection{Turbulence and the Atmospheric Boundary Layer}

The atmospheric boundary layer (ABL) is lowest layer of the atmosphere, where the atmosphere interacts directly with the underlying surface. The atmospheric flow in the $\mathrm{ABL}$ is dominated by turbulence, the small-scale air motion characterized by irregular changes in wind, temperature, and moisture. The time scale of these motions typically varies from less than a second to tens of minutes. There are cases where larger turbulent eddies can develop driven by the surface dynamic and thermodynamic forcing. Although these large eddies often organize into coherent circulations with well-defined structures (Christen et al. 2007), their movements are still chaotic and it is impossible to predict their life-cycle deterministically. As a result, turbulent motion has to be described statistically.

Atmospheric turbulence is important because turbulent mixing provides a mechanism to exchange energy, moisture, and momentum between the atmosphere and the underlying surface. The sensible and latent heating transported by atmospheric turbulence from surface to the atmosphere is a major forcing that drives atmospheric motion and weather, including tropical cyclones in the tropics and sub-tropics. This vertical transport process can be schematically illustrated by Figure 1.1 in which the sensible heat flux is used as an example. The thick solid curve represents the vertical profile of mean potential temperature. The two points A and B in the figure are subjected to an arbitrary turbulent eddy, which causes the air particle at A to gain downward motion (i.e., $w^{\prime}<0$ ). Once the particle moves downward, it becomes colder than the assumed stratified environment in which the eddy is embedded to result in a cold 
potential temperature perturbation (i.e., $\theta^{\prime}<0$ ). From this sequence, a positive sensible heat flux will be generated, i.e., $w^{\prime} \theta^{\prime}>0$. Concurrently, the air particle at $\mathrm{B}$ subjected to the same eddy will gain an upward motion (i.e., $w^{\prime}>0$ ) and a warm perturbation with respect to the mean (i.e., $\theta^{\prime}>0$ ) as it moves to a new environment. This sequence will also cause a positive sensible heat flux $w^{\prime} \theta^{\prime}>0$. Thus, a net positive sensible heat flux will be generated by this eddy despite the fact that the atmospheric perturbations created by this eddy at two sides are off-phase. This relationship for $w^{\prime}$ and $\theta^{\prime}$ will hold true independently of the size and orientation of eddies. Therefore, in this particular scenario the net result will be a positive sensible heat flux under the assumed environmental stratification. For the same reason, in a stably stratified atmosphere in which the mean potential temperature increases with height, a negative sensible heat flux will be generated. This framework applies to the vertical transport of moisture and momentum as well.

Quantitatively, the vertical turbulent transport of heat, moisture, and momentum is measured by the covariance between the vertical velocity perturbation and perturbations of potential temperature $(\theta)$, water vapor mixing ratio $(q)$, and horizontal wind components $(u$ and $v)$, i.e., $\overline{w^{\prime} \theta^{\prime}}, \overline{w^{\prime} q^{\prime}}, \overline{w^{\prime} u^{\prime}}$ and $\overline{w^{\prime} v^{\prime}}$, known as kinematic sensible heat flux, moisture flux, and momentum flux, where overbar and prime indicate the mean and the perturbation with respect to the mean.

Although the concept of vertical transport induced by turbulence is simple, an appropriate determination of turbulent fluxes of heat, moisture, and momentum is one of the major challenges in meteorology since an accurate calculation of covariance requires 
a detailed information of the chaotic turbulent eddies that span over a spectrum of temporal and spatial scales. Unfortunately, this information cannot be obtained by the convectional meteorological observations and numerical weather forecasting models. It can be obtained only by the high resolution meteorological instruments that are capable of recording the high frequency perturbations associated with turbulent eddies. Thus, a central problem of boundary layer meteorology is to search for a method that can appropriately determine turbulent fluxes using conventional meteorological measurements or variables predicted by numerical weather forecasting models.

\subsection{The Bulk Transfer Parameterization of Surface Turbulent Fluxes}

It has long been recognized that the turbulent fluxes are nearly constant in the lowest layer of ABL (approximately $30-50 \mathrm{~m}$ above the surface depending on the wind shear and static stability), a layer known as constant flux layer or surface layer (SL) in literature (e.g., Stull 1988). The constant flux assumption allows the turbulent fluxes to be related to the mean meteorological variable at a given height in the SL using the socalled bulk transfer parameterization in the form of

$$
\left\{\begin{array}{c}
u_{*}^{2}=C_{D} \bar{U}^{2} \\
\overline{w^{\prime} \theta^{\prime}}{ }_{s}=-C_{H} \bar{U}\left(\bar{\theta}-\theta_{G}\right) \\
\overline{w^{\prime} q^{\prime}}{ }_{s}=-C_{Q} \bar{U}\left(\bar{q}-q_{G}\right)
\end{array}\right.
$$

where $u_{*}=\left({\overline{u^{\prime} w^{\prime}}}^{2}+{\overline{v^{\prime} w^{\prime}}}^{2}\right)^{1 / 4}$ is the friction velocity. $\bar{U}, \bar{\theta}$, and $\bar{q}$ represent the wind speed, potential temperature, and water vapor mixing ratio at the reference height, typically $10 \mathrm{~m}$ above the surface. $\theta_{G}$ and $q_{G}$ are the surface "skin" potential 
temperature and water vapor mixing ratio, which are typically taken as the values a few centimeters above the ground surface (Stull 1988). However, since there is a sharp gradient of variables close to the surface, how to appropriately define and measure "skin" potential temperature and moisture is still debatable. The variables $C_{D}, C_{H}$ and $C_{Q}$ are the dimensionless exchange coefficients for momentum, heat, and moisture, and are often called the drag coefficient, Stanton number, and Dalton number, respectively in the relevant literature. Previous studies (e.g., Mahrt et al. 2001, Garratt 1977, Grachev et al. 2001) show that exchange coefficients exhibit a strong dependence on wind speed and atmospheric stability. Although simple and physically straightforward, the bulk transfer model itself is not closed. In order to calculate surface turbulent fluxes using Eq.1.1, exchange coefficients $C_{D}, C_{H}$ and $C_{Q}$ need to be determined externally.

The most common method used to close the bulk transfer parameterization is the well-known Monin-Obukhov similarity (MOS) theory. On the basis of the assumption of constant fluxes in the SL and using Buckingham $\pi$ theorem of dimensional analysis, Monin and Obukhov (1954) was able to derive a relationship between the vertical gradient of mean properties in the SL and surface turbulent fluxes as,

$$
\begin{aligned}
& \frac{\kappa z}{u_{*}} \frac{\partial \bar{u}}{\partial z}=\phi_{m}(\varsigma), \\
& \frac{\kappa z}{\theta_{*}} \frac{\partial \bar{\theta}}{\partial z}=\phi_{h}(\varsigma), \\
& \frac{\kappa z}{q_{*}} \frac{\partial \bar{q}}{\partial z}=\phi_{q}(\varsigma),
\end{aligned}
$$


where $\kappa$ is the Von-Karman constant. $\theta_{*}=\frac{\overline{w^{\prime} \theta^{\prime}}}{u_{*}}$ and, $q_{*}=\frac{\overline{w^{\prime} q^{\prime}}}{u_{*}}$ are the temperature scale and moisture scale, respectively. $\varsigma=\frac{z}{L}$ is the ratio of height $z$ to the MoninObukhov length defined as $L=\frac{-u_{*}^{3} \overline{\theta_{v}}}{\kappa g \overline{w^{\prime} \theta_{v}^{\prime}}}$, where $\theta_{v}$ is the virtual potential temperature. $\phi_{m}, \phi_{h}$ and $\phi_{q}$ are the dimensionless functions of atmospheric stability parameter, which cannot be obtained by the dimensional analysis itself but must be determined empirically using observations.

The most recognized empirical dimensionless stability functions used today are those obtained by Businger et al. (1971) based on results from the famous Kansas field experiment in the form of:

$$
\begin{gathered}
\begin{cases}\phi_{m}=(1-16 \varsigma)^{-1 / 4} & \text { for } \varsigma<0 \\
\phi_{m}=1+5 \varsigma & \text { for } \varsigma>0\end{cases} \\
\begin{cases}\phi_{h}=\phi_{q}=(1-16 \varsigma)^{-1 / 2} & \text { for } \varsigma<0 \\
\phi_{h}=\phi_{q}=1+5 \varsigma & \text { for } \varsigma>0\end{cases}
\end{gathered}
$$

Integrating Eqs. 1.2 - 1.4 using Eqs.1.5-1.6, one may obtain the vertical profiles of mean variables in the SL:

$$
\left\{\begin{array}{c}
\bar{u}=\frac{u_{*}}{\kappa}\left[\ln \left(\frac{z}{z_{0}}\right)-\Psi_{m}(\varsigma)\right] \\
\bar{\theta}-\theta_{G}=\frac{\theta_{*}}{\kappa}\left[\ln \left(\frac{z}{z_{0 \theta}}\right)-\Psi_{h}(\varsigma)\right], \\
\bar{q}-q_{G}=\frac{q_{*}}{\kappa}\left[\ln \left(\frac{z}{z_{0 q}}\right)-\Psi_{q}(\varsigma)\right]
\end{array}\right.
$$

where 


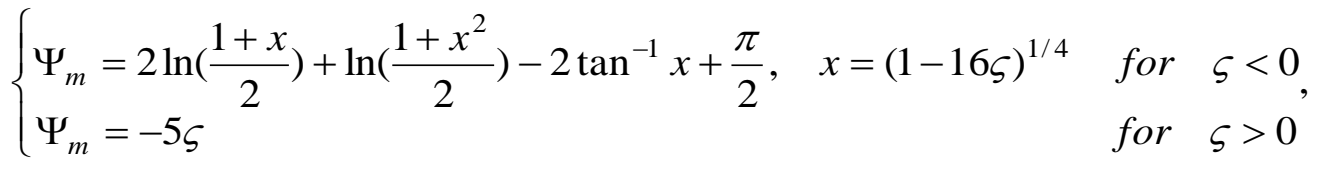

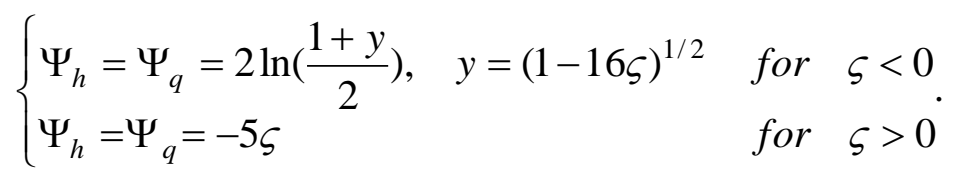

$z_{0}, z_{0 \theta}$ and $z_{0 q}$ are the surface aerodynamic roughness and thermal roughness for wind, potential temperature, and water vapor, respectively.

Combining the MOS formulae (Eq. 1.7) with the bulk transfer model (Eq.1.1), it yields,

$$
\left\{\begin{array}{c}
C_{D}=\frac{\kappa^{2}}{\left[\ln \left(\frac{z}{z_{0}}\right)-\Psi_{m}(\varsigma)\right]^{2}} \\
C_{H}=\frac{\kappa^{2}}{\left[\ln \left(\frac{z}{z_{0}}\right)-\Psi_{m}(\varsigma)\right] \cdot\left[\ln \left(\frac{z}{z_{0 \theta}}\right)-\Psi_{h}(\varsigma)\right]} \\
C_{Q}=\frac{\kappa^{2}}{\left[\ln \left(\frac{z}{z_{0}}\right)-\Psi_{m}(\varsigma)\right] \cdot\left[\ln \left(\frac{z}{z_{0 q}}\right)-\Psi_{q}(\varsigma)\right]}
\end{array}\right.
$$

Eqs. 1.1 and 1.10 provides a practical way to calculate surface turbulent fluxes using mean meteorological variables available in convectional observations and numerical simulations. To date, this method of determining surface turbulent fluxes parametrically has been widely used in all applications in geoscience from local forest/agriculture problems, weather forecasting, to global climate researches. Arguably, MOS may be considered one of the most successful theories not only in turbulence research (Wang and Bras 2009) but also in atmospheric sciences in general. 
However, a key component of the method, the stability function (Eqs. 1.5 and 1.6 or Eqs. 1.8 and 1.9) was obtained nearly 50 years ago based on the results from the Kansas field experiment on homogeneous wheat fields. Evidence has shown that turbulence motions, in particular the energy containing eddy, are strongly flow-dependent and subjected to surface conditions. Thus, it remains to be a scientific question whether those relations obtained in fair weather conditions on flat homogeneous wheat fields can be extended to severe weather conditions or complex inhomogeneous surface conditions or ocean surface. In this thesis study, I developed a method that can provide an appropriate evaluation the parameterization of surface turbulent fluxes of heat, moisture, and momentum using in-situ observations. The main objective is to revisit the surface layer turbulent flux parameterization commonly used in various applications in geosciences and provide an insight for parameterization improvement. 


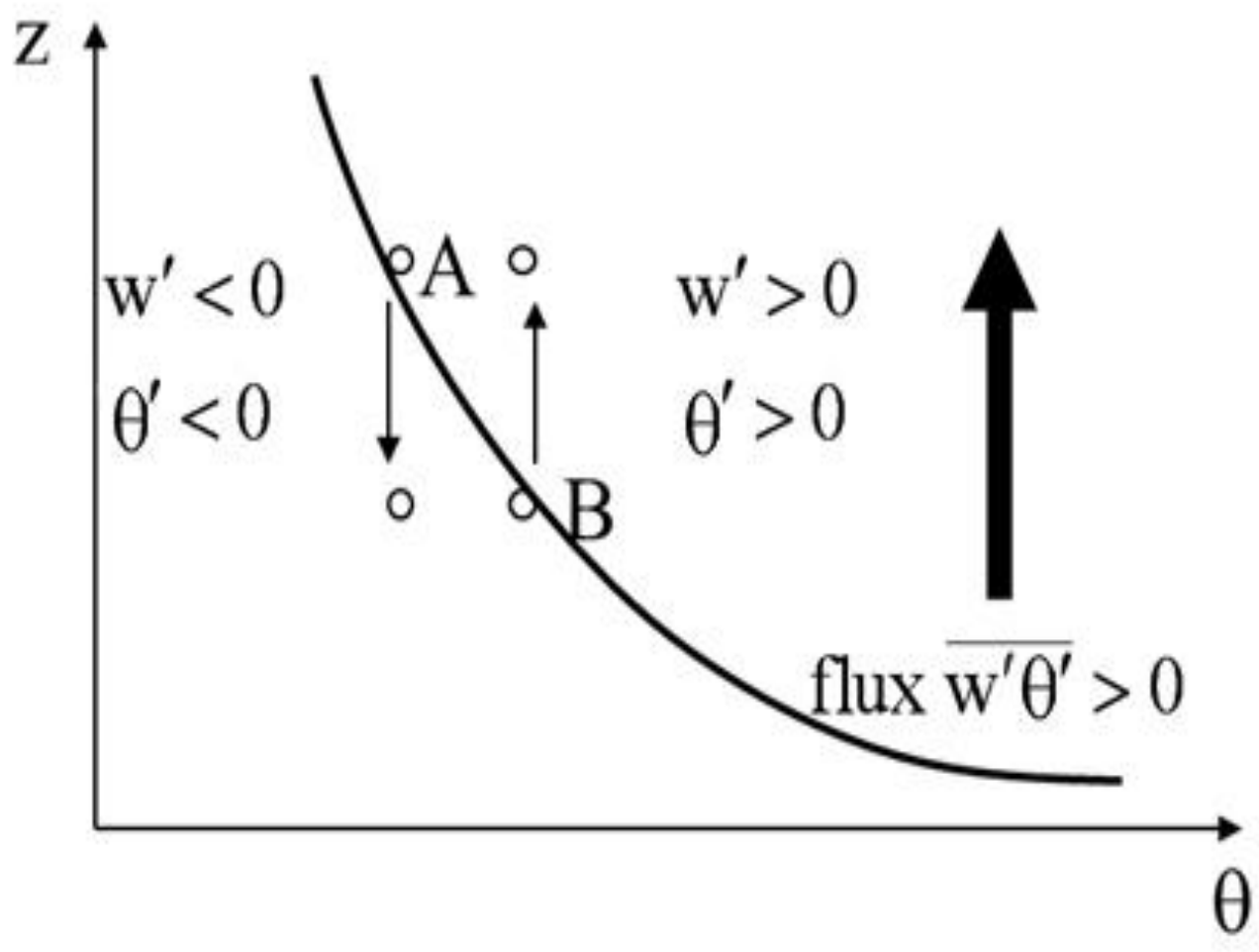

Fig. 1.1 Schematic illustration of the vertical heat fluxes induced by turbulent eddies. The thick curve indicates the vertical profile of mean potential temperature. Points A and B are subjected to an eddy circulation. Overbar and prime indicate the mean and the deviation from the mean.

Figure 1 Schematic illustration of the vertical heat fluxes induced by turbulent eddies. The thick curve indicates the vertical profile of mean potential temperature. Points A and B are subjected to an eddy circulation. Overbar and prime indicate the mean and the deviation from the mean. 


\section{Chapter II: METHODOLOGY IN EVALUATION OF SURFACE FLUX PARAMETERIZATION}

\subsection{Scientific Questions}

Recent studies show that the surface turbulent transport processes can deviate significantly from the theoretical predictions by MOS and bulk transfer model (e.g., Zhu and Furst 2013, Donelan et al. 2005). The problem can be clearly illustrated by Figure 2.1, which shows the drag coefficients in near neutral conditions $\left(C_{D N}\right)$ as a function of wind speed obtained from multiple independent field experiments over land. Although $C_{D N}$ from different field experiments shows fairly large scatter, possibly because of the large differences in surface conditions in these experiments, all the data show a clear trend of $C_{D N}$ increasing with a decrease in wind speed. However, the theoretical prediction of drag coefficients in neutral conditions according to Eq. 1.10 is a constant independent of wind speed. On the basis of their analyses, Zhu and Furst (2013) argued that such an inconsistency between observations and theoretical predictions cannot be explained by the possible increase in effective aerodynamic roughness with a decrease in wind speed because of the enhanced viscous effects and reduced the streamlining of surface obstacles proposed by Mahrt et al (2001). Rather, it reflects the internal change in turbulent energy cascade with wind speed, which is not considered in the classic MOS framework. Although Zhu and Furst (2013) focused only on the problems in the near neutral conditions, their findings suggests that there is a need to reevaluate the surface flux parameterization formulated by MOS and bulk transfer model in the stratified boundary layer as well. With present-day advances in measuring turbulence, the time is 
right to provide comprehensive evaluation of surface flux parameterization using in-situ high-resolution turbulent data, which has a much higher accuracy than that of 50 years ago.

From Eq. 1.1, the exchange coefficients can be calculated directly from observations provided that the mean meteorological variables are known and turbulent fluxes can be quantified by the eddy correlation method, which, then, can be compared with the theoretically derived exchange coefficients via Eq. 1.10 to provide an evaluation of surface flux parameterization. However, how to accurately calculate exchange coefficients using Eq.1.1 and Eq.1.10 is not a scientifically trivial question. Observationally, there are two difficulties that practically prevent calculating accurate exchange coefficients directly from Eq.1.1 and Eq.1.10. First, to calculate the exchange coefficients for heat and moisture from observations, one needs to know the surface skin temperature, $\theta_{\mathrm{G}}$, and skin moisture, $\mathrm{q}_{\mathrm{G}}$. Strictly speaking, the skin temperature and moisture should be the one defined at the height of thermal surface roughness, which is readily seen from the derivation of the vertical profiles of mean variables in the surface layer. Measuring skin temperature and moisture is difficult not only because the surface thermal roughness itself is unknown and changes from time to time for a fixed location but also because there is a sharp gradient of temperature and moisture close to the surface. Thus, a slight change in height can result in a large bias in the skin temperature and moisture measurement. Moreover, there is also a large uncertainty in measuring skin temperature and moisture caused by surface radiation, dew, surface obstacles, and other factors. As a result, it is impossible to obtain accurate surface skin temperature and moisture measurements. 
Second, in order to calculate the theoretical exchange coefficients from Eq.1.10, the surface aerodynamic roughness and thermal roughness are needed. Over the ocean, surface roughness is function of wind speed, which has been extensively investigated in many studies (e.g., Donelan et al. 2004; Black et al. 2007). Overland, wind speed may not be a major factor because of the static nature of surface roughness elements. But surface roughness depends strongly on wind direction as a result of surface inhomogeneity. As illustrated by Fig.2.2, depending on the wind direction, turbulent flow can be substantially affected by the inhomogeneous surface roughness elements. Ridder (2010) argued that MOS, which over-simplifies the complication resulting from rough terrain, may lead to the misrepresentation of the surface layer turbulent flow and the induced vertical turbulent transport. To alleviate the problem, Ridder (2010) proposed a so-called roughness sublayer (RSL) falling in between the surface and the surface layer to account for the complication from the rough terrain.

As a consequence of the previously stated difficulties in determining surface skin temperature and moisture and surface aerodynamic and thermal roughness, it is practically impossible to provide a meaningful evaluation of theoretically derived exchange coefficients using observations. Thus, to provide an appropriate evaluation of surface flux parameterization formulated using the MOS and bulk transfer model, innovative methods need to be developed.

\subsection{A new framework for evaluating surface flux parameterization}

As discussed in the previous section, an accurate calculation of exchange coefficients using Eqs. $1.1 \& 1.10$ from observations requires not only high temporal 
resolution meteorological data from which the turbulent fluxes can be quantified using eddy correlation method but also the information of surface aerodynamic roughness and thermal roughness and accurate measurements of surface skin temperature and moisture from which the thermal difference between the surface and air above it can be adequately determined. While the former is relatively easy to obtain owning to the advanced technology in sonic anemometer and gas analyzer, the latter is difficult to obtain since no method so far is available that can be used to accurately determine surface aerodynamic/thermal roughness and surface skin temperature and moisture. In this study, a novel method, which does not require surface roughness and skin temperature and moisture but can provide an appropriate evaluation of surface flux parameterization, has been proposed. The physical base and details of this method are presented as follows.

Under the assumption of constant flux in the SL, the vertical profiles of mean variables derived from MOS (i.e., Eq.1.7) can be applied to two heights, $z_{1}$ and $z_{2}$ respectively in the SL,

$$
\begin{gathered}
\left\{\begin{array}{c}
\bar{u}_{1}=\frac{u_{*}}{\kappa}\left[\ln \left(\frac{z_{1}}{z_{0}}\right)-\Psi_{m}\left(\frac{z_{1}}{L}\right)\right] \\
\bar{\theta}_{1}-\theta_{G}=\frac{\theta_{*}}{\kappa}\left[\ln \left(\frac{z_{1}}{z_{0 \theta}}\right)-\Psi_{h}\left(\frac{z_{1}}{L}\right)\right], \\
\bar{q}_{1}-q_{G}=\frac{q_{*}}{\kappa}\left[\ln \left(\frac{z_{1}}{z_{0 q}}\right)-\Psi_{q}\left(\frac{z_{1}}{L}\right)\right]
\end{array}\right. \\
\left\{\begin{array}{c}
\bar{u}_{2}=\frac{u_{*}}{\kappa}\left[\ln \left(\frac{z_{2}}{z_{0}}\right)-\Psi_{m}\left(\frac{z_{2}}{L}\right)\right] \\
\bar{\theta}_{2}-\theta_{G}=\frac{\theta_{*}}{\kappa}\left[\ln \left(\frac{z_{2}}{z_{0 \theta}}\right)-\Psi_{h}\left(\frac{z_{2}}{L}\right)\right], \\
\bar{q}_{2}-q_{G}=\frac{q_{*}}{\kappa}\left[\ln \left(\frac{z_{2}}{z_{0 q}}\right)-\Psi_{q}\left(\frac{z_{2}}{L}\right)\right]
\end{array}\right.
\end{gathered}
$$


To obtain Eqs. 2.1-2.2, we have assumed that at an instant of time the turbulent flow at $z_{1}$ and $z_{2}$ experiences the same surface roughness and stability measured by MoninOubhkov length. Subtracting one from the other, it yields,

$$
\left\{\begin{array}{l}
\bar{U}_{z_{2}}-\bar{U}_{z_{1}}=\frac{u_{*}}{\kappa}\left[\ln \left(\frac{z_{2}}{z_{1}}\right)-\Psi_{m}\left(\frac{z_{2}}{L}\right)+\Psi_{m}\left(\frac{z_{1}}{L}\right)\right] \\
\bar{\theta}_{z_{2}}-\bar{\theta}_{z_{1}}=\frac{\theta_{*}}{\kappa}\left[\ln \left(\frac{z_{2}}{z_{1}}\right)-\Psi_{h}\left(\frac{z_{2}}{L}\right)+\Psi_{h}\left(\frac{z_{1}}{L}\right)\right], \\
\bar{q}_{z_{2}}-\bar{q}_{z_{1}}=\frac{q_{*}}{\kappa}\left[\ln \left(\frac{z_{2}}{z_{1}}\right)-\Psi_{h}\left(\frac{z_{2}}{L}\right)+\Psi_{h}\left(\frac{z_{1}}{L}\right)\right]
\end{array}\right.
$$

Following the basic concept of bulk transfer model and Eq.1.1, one may represent turbulent fluxes in terms of the difference of mean variables between the two heights as,

$$
\left\{\begin{array}{c}
u_{*}^{2}=C_{D a}\left(\bar{U}_{z_{2}}-\bar{U}_{z_{1}}\right)^{2} \\
\overline{w^{\prime} \theta^{\prime}}{ }_{s}=-C_{H a}\left(\bar{U}_{z_{2}}-\bar{U}_{z_{1}}\right)\left(\bar{\theta}_{z_{2}}-\bar{\theta}_{z_{1}}\right), \\
\overline{w^{\prime} q^{\prime}}{ }_{s}=-C_{Q a}\left(\bar{U}_{z_{2}}-\bar{U}_{z_{1}}\right)\left(\bar{q}_{z_{2}}-\bar{q}_{z_{1}}\right)
\end{array}\right.
$$

where $C_{D a}, C_{H a}$ and $C_{Q a}$ are the adjusted dimensionless exchange coefficients for momentum, heat, and moisture, respectively. Combine Eq. 2.3 and with Eq. 2.4, it yields,

$$
\left\{\begin{array}{c}
C_{D a}=\frac{\kappa^{2}}{\left[\ln \left(\frac{z_{2}}{z_{1}}\right)-\Psi_{m}\left(\frac{z_{2}}{L}\right)+\Psi_{m}\left(\frac{z_{1}}{L}\right)\right]^{2}} \\
C_{H a}=\frac{\kappa^{2}}{\left[\ln \left(\frac{z_{2}}{z_{1}}\right)-\Psi_{m}\left(\frac{z_{2}}{L}\right)+\Psi_{m}\left(\frac{z_{1}}{L}\right)\right] \cdot\left[\ln \left(\frac{z_{2}}{z_{1}}\right)-\Psi_{h}\left(\frac{z_{2}}{L}\right)+\Psi_{h}\left(\frac{z_{1}}{L}\right)\right]} \\
C_{Q a}=\frac{\kappa^{2}}{\left[\ln \left(\frac{z_{2}}{z_{1}}\right)-\Psi_{m}\left(\frac{z_{2}}{L}\right)+\Psi_{m}\left(\frac{z_{1}}{L}\right)\right] \cdot\left[\ln \left(\frac{z_{2}}{z_{1}}\right)-\Psi_{q}\left(\frac{z_{2}}{L}\right)+\Psi_{q}\left(\frac{z_{1}}{L}\right)\right]}
\end{array}\right.
$$


The adjusted exchange coefficients $C_{D a}, C_{H a}$ and $C_{Q a}$ can be readily calculated from observations directly via Eq. 2.4 provided that observations are available at $z_{1}$ and $z_{2}$. They can be also calculated from Eq. 2.5 derived from MOS and the basic concept of bulk transfer model. Thus, the comparison between the observationally and theoretically determined coefficients $\left(C_{D a}, C_{H a}\right.$ and $C_{Q a}$ ) provides an objective evaluation of surface turbulent flux parameterization although the computed coefficients are not the standard exchange coefficients used in various applications in geosciences. The advantage of the new proposed method is that the uncertainties associated with surface aerodynamic and thermal roughness and surface skin temperature and moisture, which can substantially affect the accuracy of evaluation, are excluded. It should be emphasized that this method is not designed to evaluate the exchange coefficients themselves but the flux parameterization formulated by MOS and bulk transfer model. Eqs. 2.4-25, thus, provide objective evaluation framework for surface flux parameterization, and it can be readily applied to any surface and weather conditions as long as high-temporal resolution observations are available at two heights in the SL. 


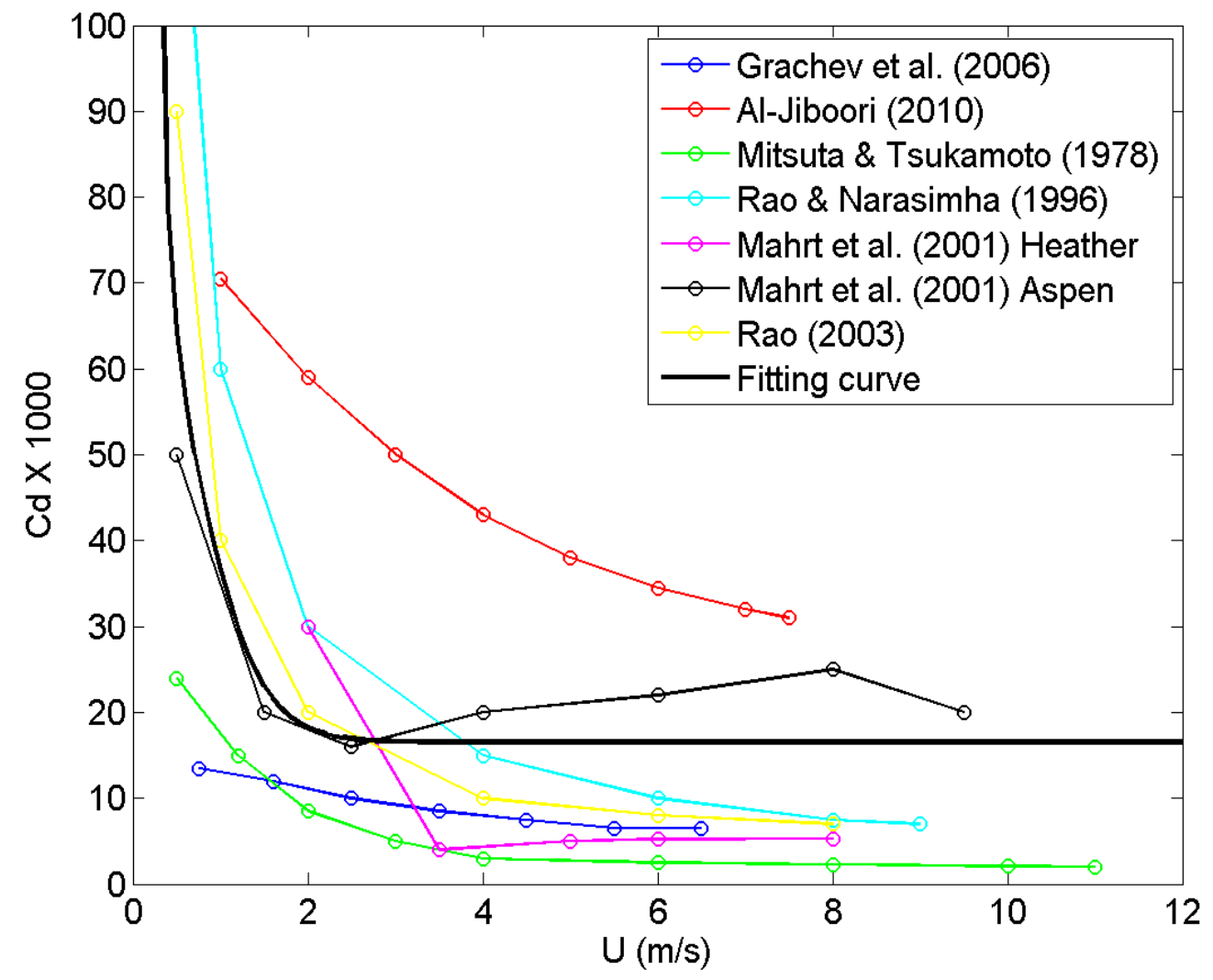

Fig. 2.1: Drag coefficients in near neutral conditions $\left(C_{D N}\right)$ against wind speed obtained from multiple independent field experiments over land (After Zhu and Furst, 2013). 

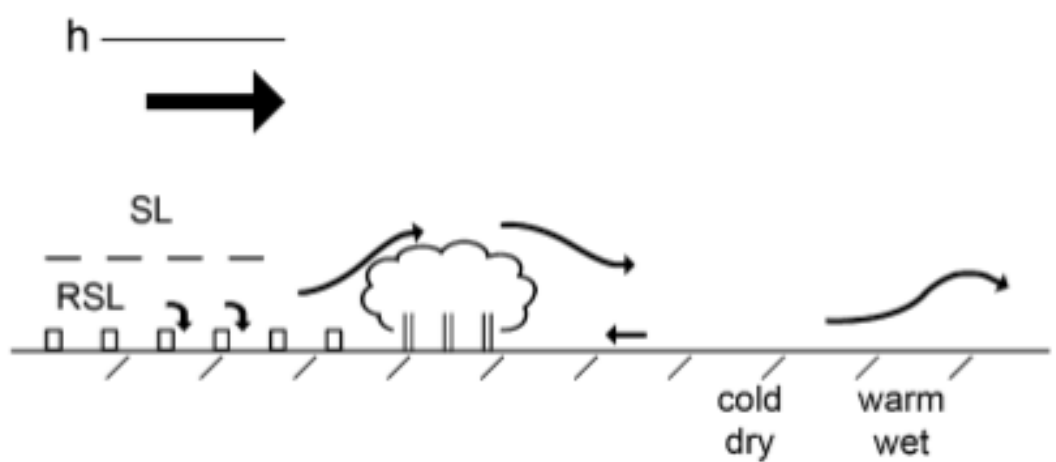

Fig. 2.2: Schematic illustration of scenarios of time-averaged turbulent motion over a rough terrain. This figure is not drawn to real scale. H, SL, and RSL are the boundary layer depth, surface layer, and roughness surface layer (after Mahrt 2001). 


\section{Chapter III: INSTRUMENTATION, OBSERVATION, AND DATA PROCESSING}

\subsection{Instrumentation}

The observational platform used in this study is the International Hurricane Research Center (IHRC) 10-meter Portable Weather Tower (PWT). The tower and trailer are shown in Fig. 3.1. Tower located instrumentation systems have been widely used in various other studies in the past to capture the rapidly changing turbulent flow within the SL. The PWT was originally constructed during the Florida Coastal Monitoring Program (FCMP), with the intent for the tower to be used in the studying of tropical cyclones as they made landfall. To that end, the PWT was built to be as resilient as possible to the strong winds of potential tropical cyclones. It can withstand up to $90 \mathrm{~ms}^{-1}$ wind gusts that often correspond to Category 5 tropical cyclones on the Saffir-Simpson Hurricane Wind Scale and weighs approximately $7000 \mathrm{lbs}$. When the PWT's mast is fully raised, its height is 10 meters. The tower mast is fixed onto a trailer that also it to be towed to potential tropical cyclone landfall positions, or where research needs dictate it to be. The PWT has housing for three oversized $12 \mathrm{~V}$ batteries that power it when there is no other access to power on site. Thus, the PWT can be deployed in remote regions without power access and can continue to function without power for an extended period of time. The PWT is also equipped with a generator for using in remote areas where power is not available. The PWT has had several successful deployments in the past with tropical cyclones landfalls, and at those times it was equipped with two sets of 3-D Gill propeller anemometers and an RM Young wind vane anemometer that were installed at the 5-m and 10-m instrument sites on the tower mast. 
During the past three years, the tower instrumentation has been upgraded. The Gill propeller anemometers and an RM Young wind vane anemometer have been replaced with two sets of Gill WindMaster Pro (WMP) sonic anemometers and LI-COR LI-7200 enclosed $\mathrm{CO}_{2} / \mathrm{H}_{2} \mathrm{O}$ gas analyzers at 5-m and 10-m. The Gill WMP sonic anemometer is designed to measure the three dimensional wind components: $u$, an along wind component; $v$, a cross wind component; and $w$, a vertical component. The basis for using a sonic anemometer is to measure the difference in transit time of an ultrasonic pulse transmitted between a pair of transducer heads. The transit time depends upon the speed of sound and the velocity of the air in its path. The path length between the pair of heads is already known, and when compared to a specific transit time, a velocity is measured from that result. To add on to this, the speed of sound is based on air density, which is also a function of temperature and water vapor mixing ratio. With this knowledge, it is possible to compute an equivalent atmospheric virtual temperature known as a sonic temperature, $T s$, through the sonic anemometer. Ts can be calculated via the following equation:

$$
T s=T \cdot\left(1+\left(\frac{\gamma_{v}}{\gamma_{d}}-\frac{M_{v}}{M_{d}}\right) \cdot \frac{e}{p-\left(1-\frac{M_{v}}{M_{d}}\right) \cdot e}\right),
$$

where, $T$ is the ambient temperature, $\frac{\gamma_{v}}{\gamma_{d}}$ is the ratio of specific heats of vapor and dry air, $\frac{M_{v}}{M_{d}}$ is the ratio of the molar masses for water vapor and dry air, $e$ is the water vapor pressure and $p$ is the total air pressure (Lanzinger at el. 2005). The WMP sonic 
anemometer has a maximum operating wind speed of $65 \mathrm{~ms}^{-1}$ and a data output rate of up to $32 \mathrm{~Hz}$. More information in regards to the Gill WMP can be found in Appendix A.

The LI-7200 closed path infrared gas analyzer measures a number of variables. The meteorological variables important to this study consist of: concentration density of water vapor, dew point temperature, total atmospheric pressure, and various other diagnostic values. The gas analyzer uses measurements of atmospheric trace gases by determining the absorption of an infrared light beam emitted within the optical path of the sensor. The infrared beam of light is absorbed by specific gases (e.g., $\mathrm{H}_{2} \mathrm{O}$ or $\mathrm{CO}_{2}$ ) at known wavelengths. This effect reduces the intensity of the infrared beam. The reduction is measured by the sensor as a function of the gases' molar concentration (Aubient 2012). The molar concentration of the measured gas can then be converted to other form of variables. For example, $\mathrm{H}_{2} \mathrm{O}$ would be converted over to water vapor mixing ratio by using the output variable labeled " $\mathrm{H}_{2} \mathrm{O}$ concentration density" and multiplying it by the ratio of molar masses for water vapor and dry air, $\frac{M_{v}}{M_{d}}$ (LI-COR Biosciences 2010).

The intake tube of the gas analyzer is connected to the inlet port, that keeps precipitation out of the gas analyzer. A flow module serves to keep a constant supply of air to the analyzer's optical path. The flow module is able to control the rate of flow of air and is measured in Standard Liters Per Minute (SLPM) and can be changed by the user. The flow rate was set to 14 SLPM for the observations in this study, which is the recommended level according to the LI-7200 user manual. It is also positioned in a manner that the same air is being sampled by the gas analyzer and the WMP. The LI- 
7200 allows for accurate data acquisition during precipitation events. In the past it was difficult to obtain useful observations during periods of precipitation events since precipitation would cause sensor malfunctions. While precipitation events can still pose a problem for instruments, the LI-7200 is capable of mitigating most of these issues. More information for the LI-7200 are included in Appendix A.

Near the base of the tower, there is a large white box that houses the data acquisition system for the data collected by the tower instruments. Collected data are sent from the instruments through a series of Ethernet cables, logged using software provide by LI-COR, and is saved as 15 minute tab delimited ASCII files onto the hard drive of a laptop located inside the white box. Instruments used in this study had a sampling rate of $20 \mathrm{~Hz}$. Near the rear left of the tower is a metallic, waterproof container where the three oversized $12 \mathrm{~V}$ batteries are located. These batteries powering all of the equipment on the tower

\section{2: Tower Deployment in SCOPE Field Experiment}

The data used in this study were collected during the Surfzone Coastal Oil Pathways Experiment (SCOPE), a nearshore field experiment supported by Gulf Of Mexico Research Initiative (GoMRI). The PWT was deployed at the Beasley Park, Fort Walton Beach, Florida from December $3^{\text {rd }}$ to December $20^{\text {th }} 2013$, during which there were three cold air outbreak events. These events allow us to investigate how weather systems affect turbulent transport in the SL in coastal regions. Figure 3.3 shows the tower deployment site, which is located at the longitude of $86.584613^{\circ}$ and latitude of $30.394424^{\circ}$. The PWT was set up in a small parking lot with a few trees and buildings to 
the west of it. Further to the northwest there are more trees and several small buildings. To the east and south of the tower there are several dunes with brushes on them and recreation pavilions. Figure 3.4 shows the local surface condition at the tower site. The PWT was blocked off with caution tape during the deployment to keep the general public away.

\subsection{Data processing}

\subsubsection{Eddy Correlation Method}

Montgomery (1948) proposed the eddy correlation method as a means to calculate the transport of heat, mass, and momentum induced by turbulent eddies. For this method to be appropriately applied, the frequency of data collection is recommended to be at least $10 \mathrm{~Hz}$ in order to resolve energy containing eddies with scales greater than the inertial sub-range in the turbulent spectrum. During the SCOPE experiment, the meteorological variables were collected at $20 \mathrm{~Hz}$, thus, the eddy correlation method is applicable.

Following the Reynolds decomposition, the instantaneous value of a generic variable can be represented as a mean and the deviation from the mean, i.e., turbulent components:

$a^{\prime}=a-\bar{a}$,

where overbar and prime indicate the mean and perturbation. In this study, the time interval used to calculate the mean is 15 minutes. We have tested several choices of time interval for turbulent statistics, such as, 10 and 20 minutes. The statistical results are nearly identical. To avoid red noise in the spectrum, a de-trending process is performed to 
remove any linear trend present. With the turbulent components determined, the variance and covariance of turbulent variables are, then, computed as:

$$
\begin{aligned}
& \overline{a^{\prime 2}}=\frac{1}{N} \sum_{i=0}^{N-1} a_{i}^{\prime 2}, \\
& \overline{a^{\prime} b^{\prime}}=\frac{1}{N} \sum_{i=0}^{N-1} a_{i}^{\prime} b_{i}^{\prime} .
\end{aligned}
$$

Applying Eqs. 3.3-3.4 to corresponding variables, kinematic momentum, heat and moisture fluxes, $\overline{u^{\prime} w^{\prime}}, \overline{v^{\prime} w^{\prime}}, \overline{u^{\prime} v^{\prime}}, \overline{w^{\prime} \theta^{\prime}}$, and $\overline{w^{\prime} q^{\prime}}$, and turbulent kinetic energy (TKE), $\frac{1}{2}\left(\overline{u^{\prime 2}}+\overline{v^{\prime 2}}+\overline{w^{\prime 2}}\right)$ can be calculated.

\subsubsection{Quality Control and Assurance Procedures}

(a) Spike Removal

As a result of the influence of dust, insects, rain droplets, wildlife feces, and other unknown reasons, high resolution data recorded by WMP and LI-COR 7200 may contain erroneous data points. Thus, quality control of measurements is required to remove the erroneous data. In this study, the method proposed by Schmid et al. (2000) is used to remove the spike data values. A spike data value is defined as follows:

$\left|\chi_{i}-\bar{\chi}_{j}\right| \geq D \cdot \sigma_{j} \rightarrow$ spike

where $\chi$ represents a generic variable; the subscript $j$ denotes the $j^{\text {th }}$ averaging time period (15 $\mathrm{min}) . \sigma_{j}$ is the standard deviation of the $j^{\text {th }}$ averaging time period. The subscript $i$ denotes the $i^{\text {th }}$ observation in the $j^{\text {th }}$ averaging time period. $D$ is a discrimination factor that depends on the variable being assessed. As with the nature of high resolution $20 \mathrm{~Hz}$ measurements of temperature and water vapor versus the 3-D wind components, a values 
of $\mathrm{D}=4.0$ is assigned for temperature and moisture, and $\mathrm{D}=5.0$ is assigned for the wind velocity components.

(b) Sonic Anemometer Tilting Correction

Once the spike data points are removed, a tilt correction algorithm needs to be applied to the sonic anemometer output to correct the possible influence of tower tilting. Previous studies have highlighted that large error in estimating momentum fluxes can occur as a result of small errors that come from misalignment of turbulent wind sensors (e.g., Pond, 1968, Deacon, 1968, Kaimal and Haugen, 1969, Dyer and Hicks, 1972). These large momentum flux errors can be traced back to the cross contamination of velocities that occur in a tilted sensor. For example, fluctuations in the longitudinal components of the wind appear as vertical velocity fluctuations, and vice versa. Figure 3.5 schematically illustrates the error induced by the slope of a terrain. Tilting cannot be completely avoided because of slight slopes in terrain or the difficultly with perfectly leveling the PWT. Observations from Wilczak et al. (2001) showed that for a $1^{\circ}$ tilt in a sonic anemometer, the estimation error of momentum fluxes is typically greater than $10 \%$ under moderately unstable conditions. However, these errors can be as large as $100 \%$ under strong convective conditions.

In a right-hand local Cartesian coordinate framework, there are three degrees of freedom available that allow three rotations around the $z$-axis, the new $y$-axis, and the new $x$-axis, respectively. These rotations can be represented mathematically without generality in the form of, 


$$
\left\{\begin{aligned}
& R_{01}= {\left[\begin{array}{ccc}
\cos \alpha & \sin \alpha & 0 \\
-\sin \alpha & \cos \alpha & 0 \\
0 & 0 & 1
\end{array}\right], } \\
& R_{12}=\left[\begin{array}{ccc}
\cos \beta & 0 & \sin \beta \\
0 & 1 & 0 \\
-\sin \beta & 0 & \cos \phi
\end{array}\right], \\
& R_{23}=\left[\begin{array}{ccc}
1 & 0 & 0 \\
0 & \cos \gamma & \sin \gamma \\
0 & -\sin \gamma & \cos \gamma
\end{array}\right],
\end{aligned}\right.
$$

where $\alpha, \beta$, and $\gamma$ are the Euler angles associated with the rotations associated with $\mathrm{x}$ axis, y-axis, and z-axis. Assuming $\left(\begin{array}{lll}\bar{u}_{0} & \bar{v}_{0} & \bar{w}_{0}\end{array}\right)$ represents the mean wind vector in the sonic anemometer coordinate frame, the mean wind vector $\left(\begin{array}{lll}\bar{u}_{3} & \bar{v}_{3} & \bar{w}_{3}\end{array}\right)$ in the desired coordinate frame after three sequences of rotation can be expressed as,

$$
\left(\begin{array}{l}
\bar{u}_{3} \\
\bar{v}_{3} \\
\bar{w}_{3}
\end{array}\right)=R_{03}(\alpha, \beta, \gamma)\left(\begin{array}{l}
\bar{u}_{0} \\
\bar{v}_{0} \\
\bar{w}_{0}
\end{array}\right), \quad R_{03}(\alpha, \beta, \gamma)=R_{23}(\gamma) \cdot R_{12}(\beta) \cdot R_{01}(\alpha) .
$$

It can be shown mathematically that the fluxes of scalars and wind stress tensor after three rotations can be written as,

$$
\left(\overline{\frac{u_{3}^{\prime} x^{\prime}}{v_{3}^{\prime} x^{\prime}}}\right)=R_{03}(\alpha, \beta, \gamma)\left(\overline{\frac{\overline{u_{0}^{\prime} x^{\prime}}}{\overline{w_{0}^{\prime} x^{\prime}}}}\right)
$$

and

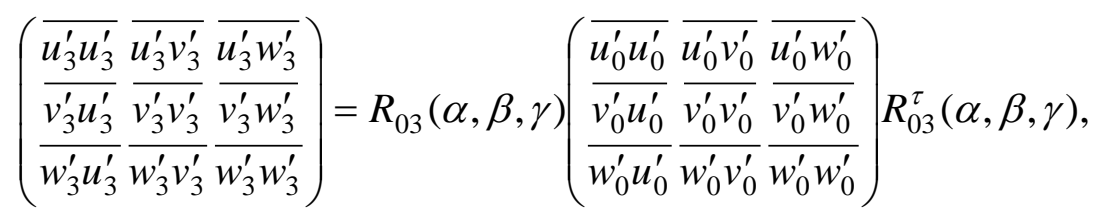

where, $R_{03}^{\tau}(\alpha, \beta, \gamma)$ is the transpose of $R_{03}(\alpha, \beta, \gamma)$. 
Using the generalized rotation transformation represented by Eqs.3.6-3.9, Tanner and Thurtell (1969) proposed a method, often known as the double rotation (DR) method, to minimize the error in estimating fluxes because of tilting by rotating the sonic anemometer coordinate into a streamwise coordinate. In the new streamwise coordinate, the $x$-axis is parallel along the mean flow and points to the down-wind direction of the mean flow. Accordingly, the $z$-axis is normal to and points away from the mean local streamline. To obtain such a desired coordinate orientation, two rotations need to be made. The first rotation involves to align $\bar{u}$ component along the mean wind direction and forces $\bar{v}=0$, which will result in the yaw angle $\alpha_{D R}$ in DR correction:

$\alpha_{D R}=\tan ^{-1}\left(\frac{\bar{v}_{0}}{\bar{u}_{0}}\right)$.

The mean wind vector after the first rotation becomes,

$$
\left(\begin{array}{l}
\bar{u}_{1} \\
\bar{v}_{1} \\
\bar{w}_{1}
\end{array}\right)=R_{01}\left(\begin{array}{l}
\bar{u}_{0} \\
\bar{v}_{0} \\
\bar{w}_{0}
\end{array}\right)
$$

The second rotation is performed in order to eliminate the mean vertical velocity $\bar{w}$. The second rotation is done under the assumption that the mean vertical velocity in the SL over a sufficiently long time period is zero. The second rotation yields the pitch angle $\beta_{D R}:$

$\beta_{D R}=\tan ^{-1}\left(\frac{\bar{w}_{1}}{\bar{u}_{1}}\right)$.

In the DR correction, only two rotations are needed, thus, the third angle $\gamma$ in Eq. 3.6 is simply zero. With the determined $\alpha_{D R}$ and $\beta_{D R}$, the corrected fluxes after the DR 
correction can be easily calculated from Eqs. 3.8-3.9. The DR method provides an optimal method to remove errors that come from a tilted sonic sensor. However, there is a potential problem with using the DR method when a mean vertical velocity that close to the surface does indeed exist. In such a case, the DR method would remove it as an error in the data assuming it as a tilting error. In this study, we carefully compared the mean vertical velocities calculated using different time intervals varying from 5 min to $30 \mathrm{~min}$. The majority of data legs do have nonzero vertical velocity, which does not appear to be realistic considering the weather conditions during the SCOPE. Therefore, the DR correction is applied to all the data collected.

\section{(c) Consistency Checks}

A series of consistency checks were made to examine if the measurements taken at the 10 and 5 meters were in agreement with each other. The first was to make sure that all the fluxes had the same sign, so that there no data points were the fluxes between the 10 and 5 meters were going in the opposite direction. An example of this would be if sensible heat flux was positive at 10 meters, but negative at 5 meters, then that point in the dataset would be removed as a result of the physical inconsistency. This is based on the basic assumption that the vertical turbulent transport cannot reverse its direction in the SL. The second consistency check is to examine if the direction of fluxes is consistent with the vertical gradient of mean meteorological variable at $10 \mathrm{~m}$ and $5 \mathrm{~m}$ altitude. For example, if the water vapor mixing ratio at $10 \mathrm{~m}$ is smaller than that at 5 meters, yet the determined latent heat flux from eddy correlation method is negative, then, the data point in the data set would be removed due to the physical inconsistency. 


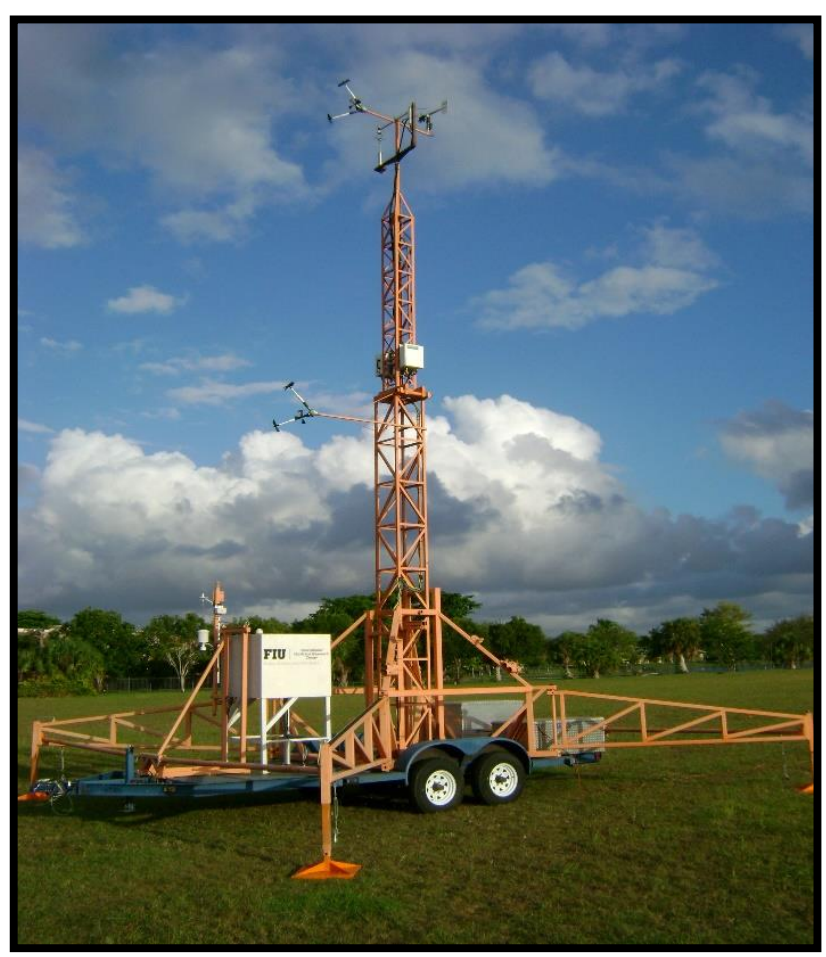

Fig. 3.1: International Hurricane Research Center (IHRC) portable meteorological tower. 


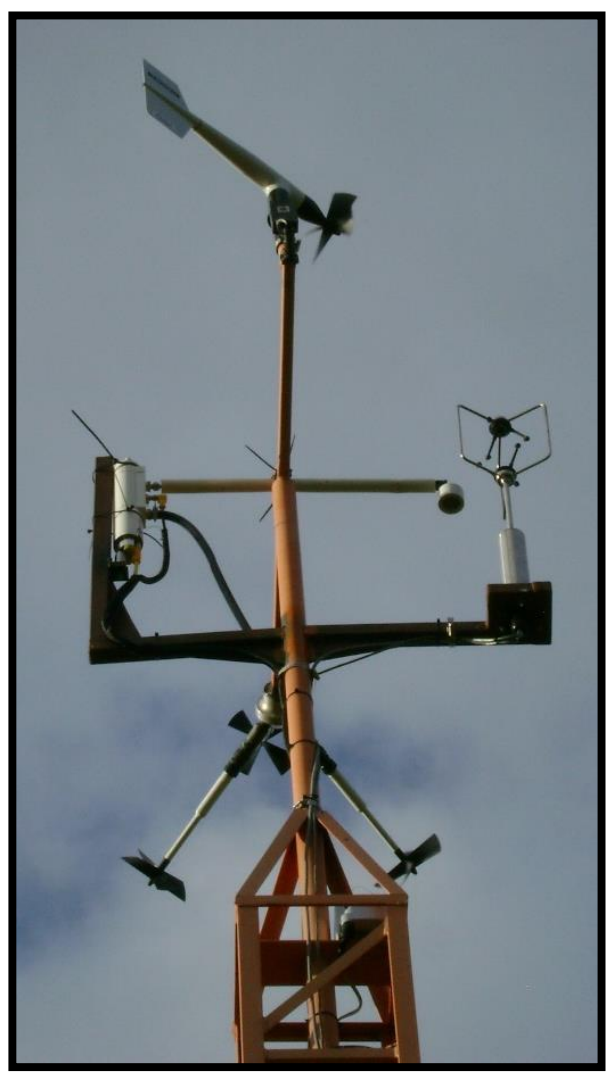

Fig. 3.2 Photo of the sonic anemometer (right) and gas analyzer (left) atop the PWT. Also shown in the photo are the pre-existing propeller anemometers (bottom) and wind vane monitor (top). 


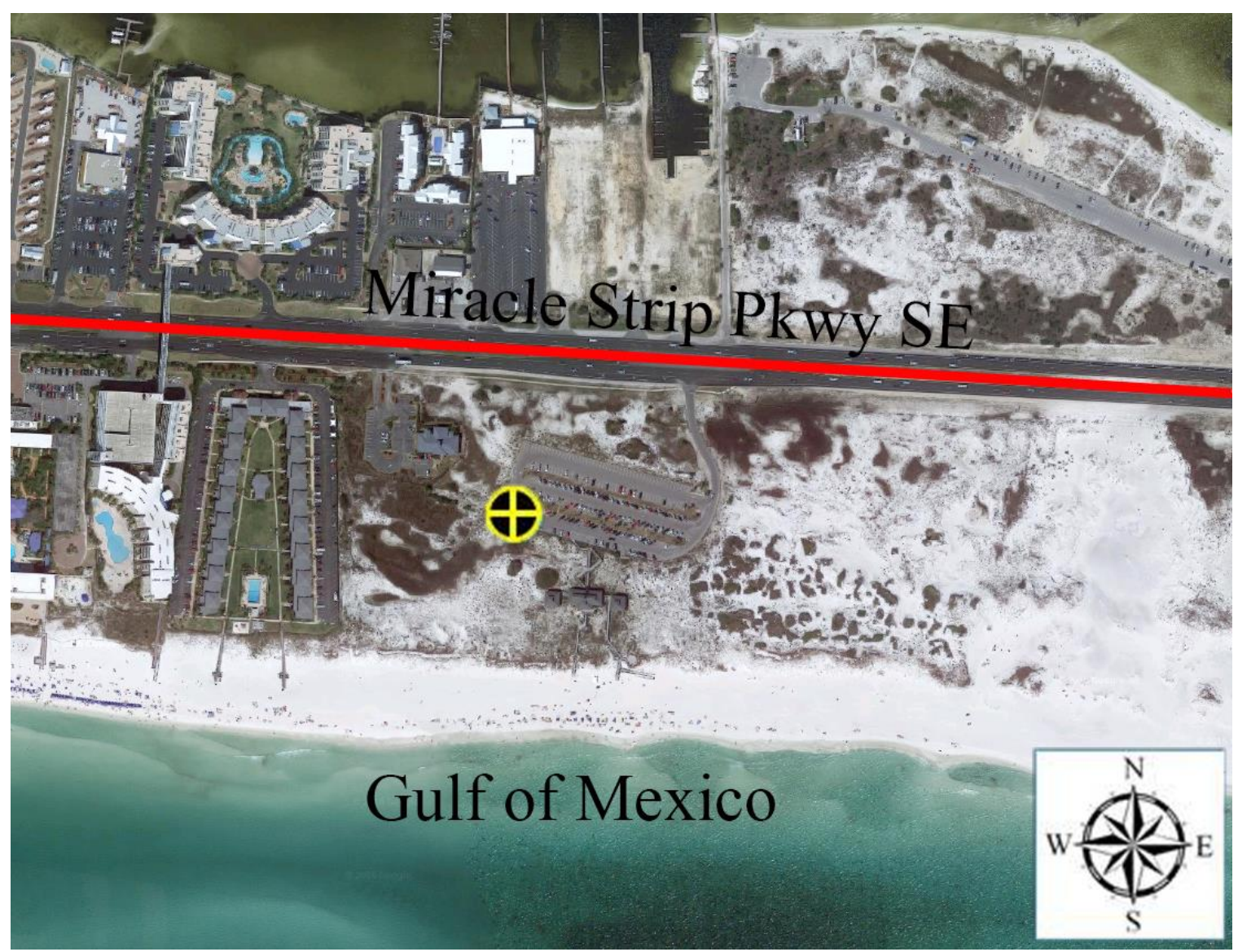

Fig. 3.3: Aerial view of PWT deployment at Beasley Park, Fort Walton Beach Florida. Small black and yellow target marks the location of PWT during the time period that it was deployed. 

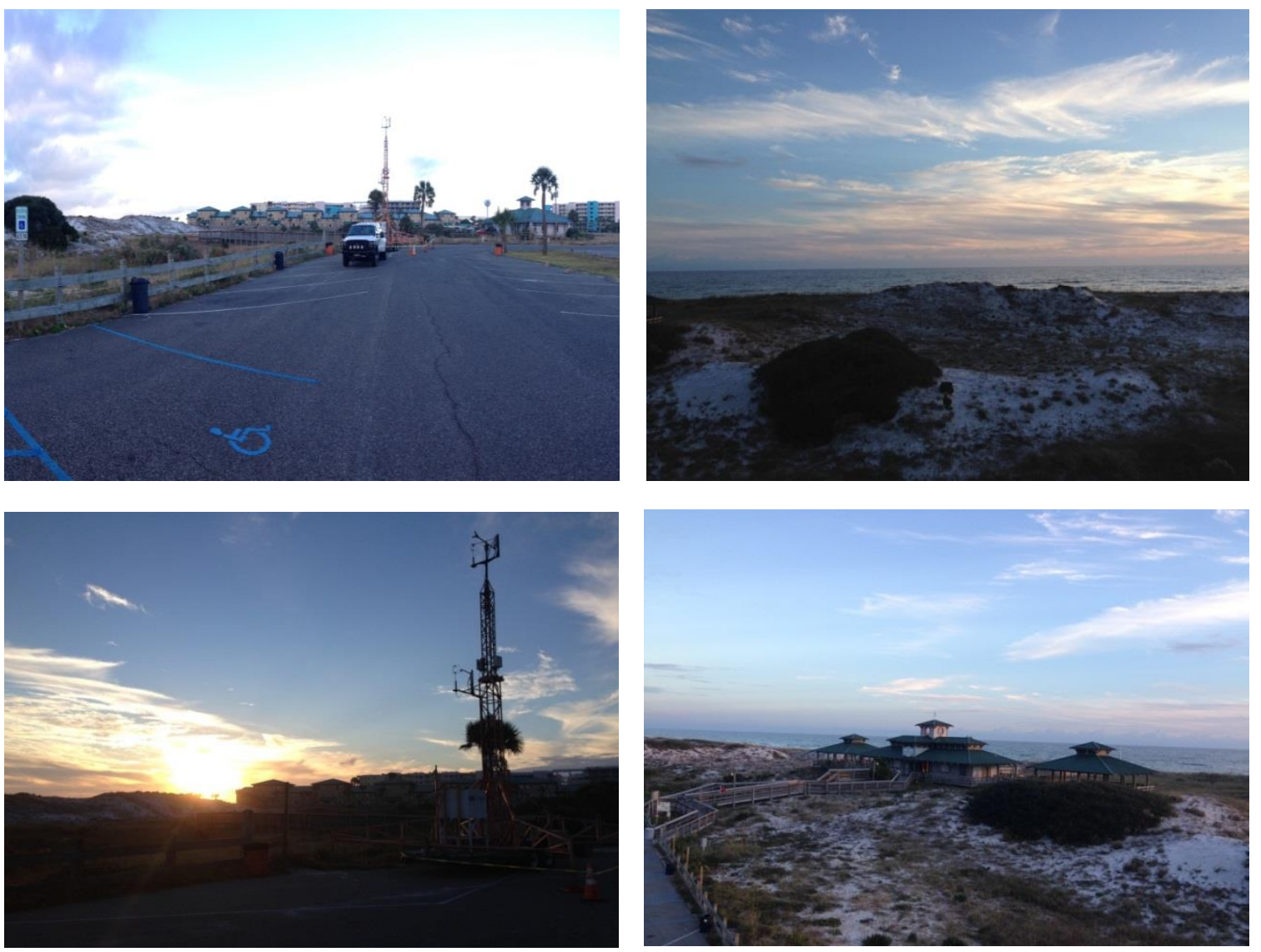

Fig. 3.4: Local surface conditions at the tower site. 

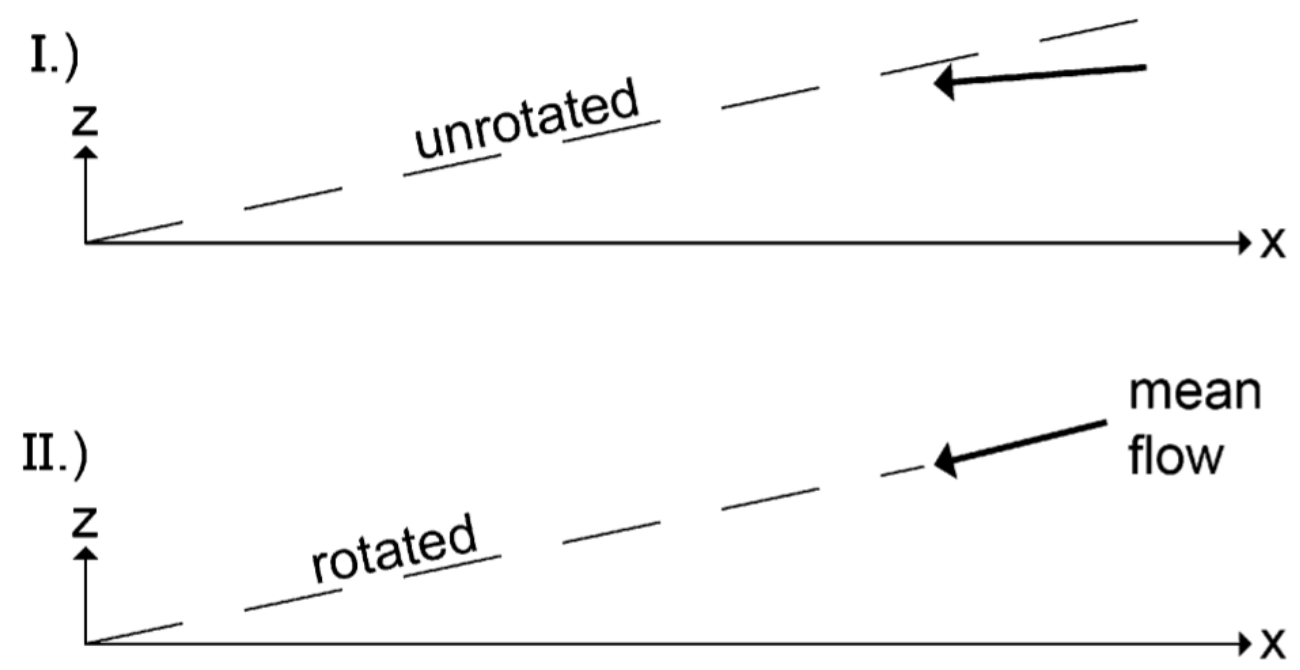

Fig. 3.5: Schematic sketch showing the error caused by misalignment of sonic anemometer (after Mahrt 2010). 


\section{Chapter IV: DATA ANALYSES AND RESULTS}

\subsection{Overview of SCOPE Tower Observations}

During the SCOPE, IHRC PWT successfully collected 3D wind components, atmospheric pressure, sonic virtual temperature, and water vapor mixing ratio at $20 \mathrm{HZ}$ at $10 \mathrm{~m}$ and $5 \mathrm{~m}$ from 11:45 UTC, December 3 to 12:00 UTC, December 20, 2013 without interruption. Figure 4.1 shows the time series of 15-min mean west-east and south-north wind components, temperature, and water vapor mixing ratio at $10 \mathrm{~m}$ and $5 \mathrm{~m}$ compared with the NCEP FNL (Final) Operational Global Analysis data at the grid point closest to the tower deployment site. The tower measurements match the FNL analyses fairly well, suggesting that the local terrain and inhomogeneity do not interrupt the large-scale flow pattern to the first order approximation. The tower observations catch 17 diurnal cycles although some of them are interrupted by the weather systems, in particular, the cold frontal passage. There were three cold outbreaks occurred during the deployment period. The first of which occurred on December $7^{\text {th }}$. However, this front would then retrograde back north to only go move southward and through Beasley Park on the $10^{\text {th }}$. A third and final cold front for the deployment period passed through the region on $14^{\text {th }}$ bringing with it some thunderstorms as well. The signature of cold frontal passage is also shown in the sudden change in wind direction and decrease of water vapor mixing ratio, which corresponds well with the temperature drop. For most of the time during the experiment, winds were coming from north and northwest associated with the cold frontal passage, but there were times when winds were coming from south and southeast. Thus, depending on the wind direction, the PWT collected the turbulent flow data over both land and ocean surface conditions. 
The time series of friction velocity, TKE, sensible heat fluxes, and latent heat fluxes of each 15-min data leg determined by the eddy correlation method at $10 \mathrm{~m}$ and 5 $\mathrm{m}$ appear in Fig. 4.2. While there are well-defined diurnal cycles shown in surface sensible heat fluxes, the time variation of surface latent heat fluxes does not exactly follow the diurnal variation of sensible heat fluxes, indicating the complication of coastal surface inhomogeneity to surface turbulent transport processes. Yet, the larger magnitude and more apparent diurnal variation of surface sensible heat fluxes than that of latent heat fluxes suggest that the surface evaporation (or latent heating of the atmosphere) is not the dominant energy source at the coast even at the beachfront so close to ocean. The dominant source of energy not coming from surface evaporation is likely caused by the fact that winds were mainly coming from inland during this experiment.

There are three large episodes of TKE shown in time series of observations. They correspond well with the cold frontal passages, however, no such signature is shown the momentum and heat fluxes, suggesting that although the perturbations generated by the cold outbreaks are an important source of TKE, they are not the efficient flux carrier since the vertical velocity perturbation associated with frontal processes do not appear to be well correlated to the perturbations of thermodynamic variables. The situation is different from the typical thermal plumes in which the perturbations of vertical velocity and conservative thermodynamic variables are well correlated. As a result of a correlation between the frontal processes and the perturbations of thermodynamic variables, in the present study we do not treat the surface fluxes associated with the frontal passages separately from those of other time periods, rather, following the classic way of boundary layer research we investigate the surface turbulent transport processes in terms of 
atmospheric stability and wind speed. This treatment of turbulent fluxes is also derived from the consideration that surface flux parameterization is developed based on the mean meteorological variables rather than specific weather events.

To examine the basic assumption of constant fluxes in the SL, Fig. 4.3 plots the fluxes and TKE of all 15-min data legs at $10 \mathrm{~m}$ against those at $5 \mathrm{~m}$. Overall, the constant flux assumption holds well for the entire dataset collected during the experiments. The results showing that the constant flux assumption holds gives us the confidence that the method for evaluating surface flux parameterized developed in section 2.2 is applicable to the turbulent data collected in the SCOPE experiment. The figure also shows that the TKE at the two levels are nearly identical. This correlation of TKE at two heights is interesting since TKE is determined not by covariance but by the variance of velocity. It suggested that the constant flux assumption of the SL may be extended to the secondorder turbulent moments in general. This assumption is confirmed by the linear relationship between TKE and square of friction velocity, which will be shown in Section 4.3. However, data spreads along the constant line are shown in all second-order turbulent moments, suggesting that there are processes that tend to break down the constant assumption in the SL. The cause of the spreading will be further investigated in Section 4.4.

\subsection{Evaluation of Surface Flux Parameterization}

In Section 2.2, I presented a method for evaluating surface flux parameterization via comparison of observationally determined and theoretically derived adjusted exchange coefficients of two levels in the SL. This proposed method does not require information of surface roughness and skin temperature and moisture, and thus, can be 
easily applied to the observations collected in this study. Figure 4.4 shows the observationally determined adjusted drag coefficients calculated by Eq. 2.4 compared with the theoretically derived adjusted drag coefficients calculated by Eq. 2.5 where for a better illustration of the data, the scale of $C_{D a}$ (y-axis) has been nonlinearly adjusted. Note that the stability parameter, the Monin-Oubhkov length, is calculated directed from its definition, $L=\frac{-u_{*}^{3} \overline{\theta_{v}}}{k g \overline{w^{\prime} \theta_{v}^{\prime}}}$ using observations, and the averaged $L$ at $10 \mathrm{~m}$ and $5 \mathrm{~m}$ is used in the theoretical calculation of $C_{D a}$. The figure clearly shows that most of the data points fall in the near neutral and weak stratification conditions. There is a large spread in the data (i.e., a substantial change in $C_{D a}$ ) shown in the stability range near $\zeta=z / L=0$, indicating that $C_{D a}$ is extremely sensitive to the stability change in the near neutral conditions. A slight change in stability can result in a large change in $C_{D a}$. In other words, a tiny error in estimating stability can lead to a large bias in determination of drag coefficient. This sensitivity is a major uncertainty in the estimation of drag coefficient and poses a great challenge in calculation of surface momentum fluxes parametrically. The theoretical prediction of $C_{D a}$ by the default MOS formula (blue curve) deviates substantially from the observed values, particularly in the unstable conditions. It fails to represent the strong dependence of $C_{D a}$ on stability in the near neutral conditions and tends to underestimate the value of $C_{D a}$. However, it is shown that a better fitting of data points can be obtained by tuning the Von-Karman constant, $\kappa$. The best fitting of the data yields the value of $k=1.1$.

The result shown in Fig. 4.4 may have important implications. It suggests that the empirical stability function obtained from the Kansas field experiment by Businger et al. 
(1971) can be extended to different surface conditions other than homogeneous wheat fields, and the improvement of surface flux parameterization may be achieved in the existing parameterization framework. However, some assumptions used by the classic MOS, such as Von-Karman constant, need to be reevaluated using extensive observations collected by more advanced instrumentation than 50 years ago. The possible ways to improve the current parameterization framework for surface fluxes will be discussed in detail in Section 4.3.

The comparison between observationally determined and theoretically derived adjusted exchange coefficient of heat, $C_{H a}$, for all 15-min data legs is shown in Fig. 4.5. Again, the observations show a substantial sensitivity of $C_{H a}$ to stability parameter in the near neutral conditions. But such a sensitivity cannot be well represented the default MOS formula. Tuning Von-Karman constant, $k$, obtains a better data fitting in the unstable range, but alone it is not enough to match the observations in the stable conditions. This result is not a surprise since intermittent turbulence in the stable conditions is notoriously difficult to represent. It turns out that a better fitting of observations points can be obtained by further tuning the parameter, $\gamma$, in the empirical stability function, $\phi_{h}=1+\gamma \frac{z}{L} ;$ for $\frac{z}{L}>0$. The best fitting of the data yields $k=0.6$ and $\gamma=650$.

Similar results are found for the adjusted exchange coefficient of moisture, $C_{Q a}$ (Fig. 4.6). The observations show a strong dependence of the adjusted coefficients on stability parameter in the near neutral conditions, and the default MOS formula is unable to represent the observed variation well. The best fitting yields $k=0.1$ and $\gamma=50$. It should 
be emphasized that in many applications including some well-known numerical models, such as, Weather Research and Forecasting (WRF) model and Hurricane WRF (HWRF) model, the exchange coefficients for heat and moisture are treated as identical. This assumption for the exchange coefficients for heat and moisture may not be appropriate according the SCOPE data. In fact, there were no fast-responding moisture observations available in the Kansas field experiment that can be used to quantify moisture fluxes using eddy correlation method. Whether the empirical stability function for heat, $\phi_{h}$, can be extended to moisture field was never discussed in the original work of Businger et al. (1971). The assumption that $\phi_{h}$ is the same as $\phi_{q}$ was made later in applications mainly due to the lack of accurate moisture data. This is because the techniques to obtain fastresponding temperature and moisture measurements are different. The former can be accurately obtained from sonic anemometer, whereas the latter relies on the gas analyzer, a technique developed in the late 90s. With the current advanced gas analyzer techniques, it is the time to revisit this issue, which will be addressed in my future research.

Figures 4.4-4.6 show that the observationally determined adjusted exchange coefficients exhibit a certain data spread. To explore what causes the spread, we grouped the same data into two categories with wind speed greater and smaller than $2 \mathrm{~ms}^{-1}$. The results are shown in Fig. 4.7, which clearly shows that the large spread of observations mainly occurs in the low-wind regime. The large spread in calm-wind conditions is likely caused by the convectively driven boundary layer in which convective plumes can generate strong non-local mixing and the strong stable boundary layer in which only intermittent turbulence may occur. Previous studies show that both of the scenarios 
appeared to be problematic for MOS. Thus, attentions need to be paid to the calm-wind conditions in future research.

\subsection{Issues Regarding Basic Assumptions of MOS}

\subsubsection{Von-Karman Constant}

In Section 4.2, the Von-Karman constant in the theoretically derived adjusted exchange coefficients was tuned to provide the best fit of the observationally determined coefficients. A question remains to be addressed here is: if such tuning of the VonKarman constant is physically robust? Historically, the MOS formula Eq. 1.2 was derived from the Buckingham Pi theorem of dimensional analysis in which $k$ needs to be treated as a constant known as Von-Karman constant. Then, it would difficult to justify a varying $k$ within the classic MOS framework. In a recent study, Zhu and Furst (2013) showed that Eq. 1.2 does not have to be obtained from a pure dimensional analysis perspective; it can be derived directly from the TKE budget equation,

$$
\frac{\partial e}{\partial t}=-\overline{u^{\prime} w^{\prime}} \frac{\partial \bar{u}}{\partial z}+\frac{g}{\theta_{0}} \overline{w^{\prime} \theta^{\prime}}-\frac{\partial \overline{w^{\prime} e}}{\partial z}-\frac{1}{\rho_{0}} \frac{\partial \overline{w^{\prime} p^{\prime}}}{\partial z}-\varepsilon_{e},
$$

where $e$ represents TKE, $p$ is pressure, and $g$ is gravity. $\theta_{0}$ and $\rho_{0}$ are ambient potential temperature and air density, respectively. The term on the left-hand side (LHS) is the local storage term. The first, second, third, fourth, and fifth terms on the right-hand side (RHS) of Eq. 4.1 are the TKE shear production term, buoyancy production term, vertical transport term, pressure correlation term, and dissipation term, respectively.

Under the assumption of constant flux $\left(\overline{w^{\prime} e}=\right.$ constant; $\overline{w^{\prime} p^{\prime}}=$ constant $)$ in the SL, for a steady neutral boundary layer flow $\left(\frac{\partial e}{\partial t}\right.$ and $\left.\overline{w^{\prime} \theta^{\prime}}=0\right)$, Eq. 4.1 reduces to, 
$-\overline{u^{\prime} w^{\prime}} \frac{\partial \bar{u}}{\partial z}=\varepsilon_{e}$

Eq. 4.2 indicates a balance between shear production and dissipation. In high order turbulent closure models (e.g., Mellor and Yamada 1974; Zeman, 1981; Deardorff 1973), the TKE dissipation is often parameterized as:

$\varepsilon_{e}=\frac{e^{3 / 2}}{\Lambda}$

where $\Lambda$ is the empirical dissipation length scale, and in the SL it is often considered as a function of height,

$\Lambda=c_{2} z$

where $c_{2}$ is an empirical coefficient. The SCOPE observations and observations collected in other field experiments show that there is a linear relationship between TKE and square of frictional velocity (Fig. 4.8), thus, TKE may be represented in terms of $u_{*}^{2}$ as,

$u_{*}^{2}=c_{1} e$,

where $c_{1}$ is a linear regression coefficient. The best fitting yields $c_{1}=0.2$. With Eqs. 4.34.5, the balance between TKE shear production and dissipation, Eq.4.2, can be rewritten as, $\frac{\eta z}{u_{*}} \frac{\partial \bar{u}}{\partial z}=1, \quad \eta=c_{1}^{3 / 2} c_{2}$

Eq. 4.6 has the exactly same format of MOS formula in neutral conditions, $\frac{\kappa z}{u_{*}} \frac{\partial \bar{u}}{\partial z}=1$.

Coefficient $\eta$ in our new parameterization framework based on the TKE budget analyses is equivalent to the Von-Karman constant in the MOS framework but it does not have to 
be a constant. Zhu and Furst (2013) showed that $\eta$ is a function of wind speed in neutral conditions, i.e., $\eta=\eta(\bar{u})$.

In the non-neutral condition, the TKE buoyancy production needs to be considered. The TKE budget equation then becomes:

$-\overline{u^{\prime} w^{\prime}} \frac{\partial \bar{u}}{\partial z}+\frac{g}{\theta_{0}} \overline{w^{\prime} \theta^{\prime}}=\varepsilon_{e}$

Normalizing Eq. 4.7 by the shear production term, yields:

$1-R_{f}=\frac{\varepsilon_{e}}{-\overline{u^{\prime} w^{\prime}} \frac{\partial \bar{u}}{\partial z}}$,

where, $R_{f}=\frac{g}{\theta_{0}} \overline{w^{\prime} \theta^{\prime}} / \overline{u^{\prime} w^{\prime}} \frac{\partial \bar{u}}{\partial z}$ is the flux Richardson number. Applying Eqs. $4.3-4.5$, and using the definition of the Monin-Obukhov length $\left(L=\frac{-u_{*}^{3} \bar{\theta}}{k g \overline{w^{\prime} \theta^{\prime}}}\right)$, Eq. 4.8 may be rewritten as,

$\frac{\eta z}{u_{*}} \frac{\partial \bar{u}}{\partial z}=1+\frac{\eta}{\kappa} \frac{z}{L}=\varphi_{m}\left(\bar{u}, \frac{z}{L}\right)$.

Eq. 4.9 is equivalent to the MOS formula, $\frac{k z}{u_{*}} \frac{\partial \bar{u}}{\partial z}=\phi_{m}\left(\frac{z}{L}\right)$. Thus, it is arguable that the MOS formula derived from the dimensional analysis may be considered as a specific case in our more generalized framework.

In neutral conditions, Zhu and Furst (2013) showed that $\eta$ can be directly estimated from the Kolmogorov's energy spectrum law. Kolmogorov first showed that in 
the inertial sub-range, the energy density per unit wave number depends only on the wave number and the rate of energy dissipation. Dimensional analysis yields:

$S(v)=\alpha \varepsilon_{e}^{2 / 3} v^{-5 / 3}$

where $v$ is the angular wave number, $S(v)$ is the energy density per unit wave number in the inertial sub-range, and $\alpha$ is the universal Kolmogorov constant. Equation 4.10 is also known as Kolmogorov's 5/3 power law. Converting Eq. 4.10 from wave number domain to frequency domain, yields:

$\varepsilon_{e}=\alpha^{-3 / 2} \frac{2 \pi f}{U}[f S(f)]^{3 / 2}$,

where to obtain Eq. 411 the relationship $v=\frac{2 \pi f}{U}$ is used, $U$ is the mean wind speed, and $S(f)$ is the energy density per unit frequency. Theoretically, the energy density of the three wind components $u, v$, and $w$ should be the same in the inertial sub-range, and this allows us to determine TKE dissipation, $\varepsilon_{e}$, based in Eq. 4.11. To validate the theoretical conclusion that wind components $u, v$, and $w$ have the same spectra in the inertial subrange, we carefully examined the turbulence spectra of the SCOPE data. As an example, Figure 4.9 shows power spectra of $u, v$, and $w$ of an arbitrary spectral lag (15 min) from the ENP data. Several features are shown in the figure. First, in the inertial sub-range, the spectra of $u, v$, and $w$ all follow the $-5 / 3$ power law nicely, indicating the good quality of the SCOPE data. Second, for large energy containing eddies, the magnitude of $w$ power spectra is substantially smaller than that of horizontal ( $u$ and $v$ ) wind power spectra. This result is consistent with previous studies (e.g., Kaimal et al. 1972; 1976; and Busch 1973). Finally, the power spectra magnitudes of the different wind components are nearly 
the same in the inertial sub-range consistent with the theoretical derivation, confirming that the energy dissipation rate can be estimated from the power spectra of horizontal and vertical wind components. As shown in Fig. 4.9, although the spread of power spectra in the inertial subrange is fairly large, there is no apparent trend. Thus, the averaged $\varepsilon_{e}$ over the frequency band in the inertial subrange, which is defined from $0.1 \mathrm{~Hz}$ to $5 \mathrm{~Hz}$ in this study, can be considered as the mean $\varepsilon_{e}$ for a specific spectrum lag (15min). Once $\varepsilon_{e}$ is determined, the coefficient $c_{2}$ can be estimated based on Eqs. 4.3-4.4. Figure 4.10 shows the estimated coefficient $\eta=c_{1}^{3 / 2} c_{2}$ as the function of wind speed for the SCOPE data in near neutral conditions $\left(0.1<\varsigma=\frac{z}{L}<0.1\right)$. It clearly shows that the coefficient $\eta=c_{1}^{3 / 2} c_{2}$ is not a constant but instead increases with the decrease of wind speed. The conclusion is that the Von-Karman constant from the dimensional analysis argument may not be really a constant in the real SL. Abandoning this assumption in the new parameterization framework developed from the TKE budget analysis is possible, and this abandonment allows for more freedom to improve surface flux parametrization. So far, we only focused on the neutral conditions. How stability affects $\eta$ and how to extend Eq. 4.9 to thermodynamic variables will be investigated in my future research.

\subsubsection{Constant Flux Assumption in the SL}

The MOS theory and the method for evaluating surface flux parameterization developed in this study are based on the constant flux assumption in the SL. However, as indicated in Fig. 4.3, there is a fairly large spread of data along the constant line, suggesting that there must be processes that tend to break down the constant flux 
assumption. Since turbulent eddies span a spectrum of scale, it is a natural question as to if all scale eddies tend to satisfy this assumption. This question may be addressed by decomposing the turbulent fluxes in terms of scales using fast Fourier Transform (FFT).

Let $w(n)$ and $\chi(n)$ be the time series of vertical velocity and a generic scalar, where $n=0,1, \ldots N-1$ are the observation points. The FFT of $w(n)$ and $\chi(n)$, then, may be written as,

$$
\begin{aligned}
& \hat{w}(k)=\frac{1}{N} \sum_{n=0}^{N-1} w(n) e^{-i \omega_{k} n}, \\
& \hat{\chi}(k)=\frac{1}{N} \sum_{n=0}^{N-1} \chi(n) e^{-i \omega_{k} n},
\end{aligned}
$$

where $k$ is the FFT frequency, and $\omega_{k}=2 \pi \frac{k}{N}, k=\left[-\frac{N}{2}: 1: \frac{N}{2}-1\right], \frac{\omega_{k}}{\Delta t}$ is the angular frequency. $\Delta t=1 / 20 \mathrm{~s}$ is the data sampling interval. The spectral energy, then, may be written as,

$$
\begin{aligned}
& E_{w}(k)=\hat{w}(k)^{*} \cdot \hat{w}(k)=|\hat{w}(k)|^{2}, \\
& E_{\chi}(k)=\hat{\chi}(k)^{*} \cdot \hat{\chi}(k)=|\hat{\chi}(k)|^{2},
\end{aligned}
$$

where superscript * denotes the complex conjugate. One may also define the cross spectrum between $w(n)$ and $\chi(n)$ as,

$$
\begin{aligned}
G_{w \chi}(k)= & \hat{w}(k)^{*} \cdot \hat{\chi}(k)=\left[\hat{w}(k)_{r}-i \hat{w}(k)_{i}\right]\left[\hat{\chi}(k)_{r}+i \hat{\chi}(k)_{i}\right] \\
& =\left[\hat{w}(k)_{r} \cdot \hat{\chi}(k)_{r}+\hat{w}(k)_{i} \cdot \hat{\chi}(k)_{i}\right]-i\left[\hat{w}(k)_{i} \cdot \hat{\chi}(k)_{r}-\hat{w}(k)_{r} \cdot \hat{\chi}(k)_{i}\right], \\
& =C_{w \chi}(k)-i Q_{w \chi}(k)
\end{aligned}
$$

where subscripts $r$ and $i$ indicate the real and imaginary parts of a complex. $C_{w \chi}(k)$ and $Q_{w \chi}(k)$ are known as the cospectrum and quadrature spectrum, respectively. The 
Parseval's theorem states that the sum of spectral energy and cospectral amplitudes over all FFT harmonics equals the variance of $w(n)$ and $\chi(n)$ and the covariance between them, respectively, i.e.,

$$
\begin{aligned}
& \sum_{k=0}^{N-1} E_{w}(k)=\overline{w^{\prime 2}} \\
& \sum_{k=0}^{N-1} E_{\chi}(k)=\overline{\chi^{\prime 2}} \\
& \sum_{l=0}^{N-1} C_{w \chi}(k, l)=\overline{w^{\prime} \chi^{\prime}}
\end{aligned}
$$

Thus, $C_{w \chi}(k)$ provides a way to decompose a vertical flux in terms of FFT harmonics. Using this method, I decomposed the momentum, sensible heat, and latent heat fluxes for all flux legs (15 min) of the SCOPE data. Figure 4.11 shows the decomposition of latent heat fluxes in terms of eddy frequencies for all flux legs $(15 \mathrm{~min})$ of the SCOPE data. Since the mean wind speed during the SCOPE was about $4 \mathrm{~m} / \mathrm{s}$, the frequency of $0.02 \mathrm{~Hz}$ approximately corresponds to a spatial scale of $200 \mathrm{~m}$. Thus, the results shown in Fig.4.11 indicate that fluxes induced by turbulence smaller than $200 \mathrm{~m}$ are nearly constant in the SL. However, large eddy circulations tend to break the constant flux assumption of MOS. Similar results are shown in momentum fluxes and sensible heat fluxes (not shown here). Since large eddies are more flow dependent and more subjected to underlying surface, we speculate that the constant flux assumption will be substantially affected by the complex terrain and mesoscale and synoptic weather systems and may not be held as well as that in fair weather condition over the homogeneous terrain. The 
unique characteristics of large eddies poses a great challenge for including their contribution surface flux parameterization. 
(a) $\cup(w-e)(m / s)$

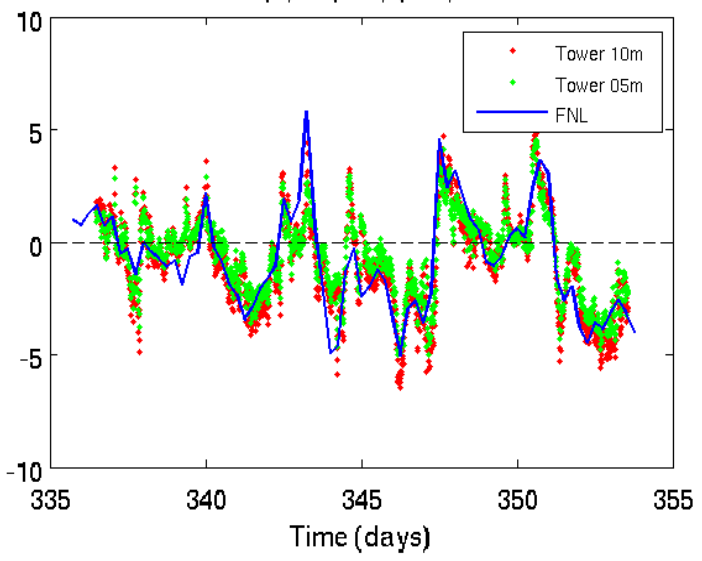

(c) Temperature (K)

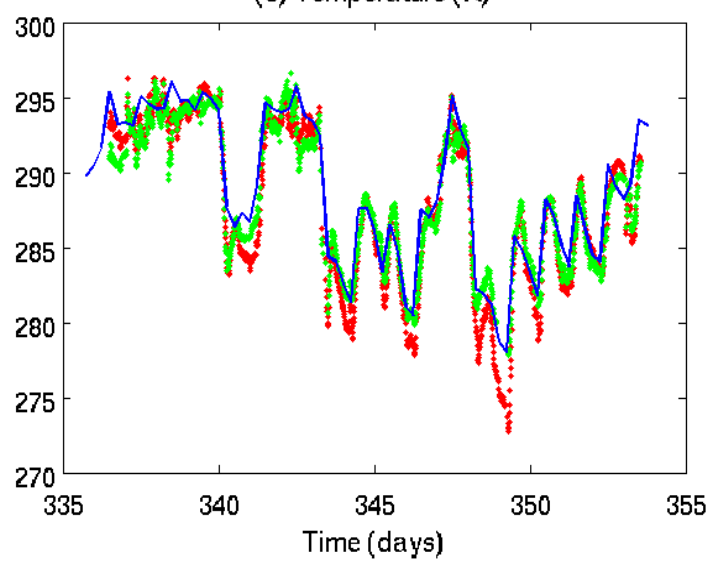

(b) $V(s-n)(m / s)$

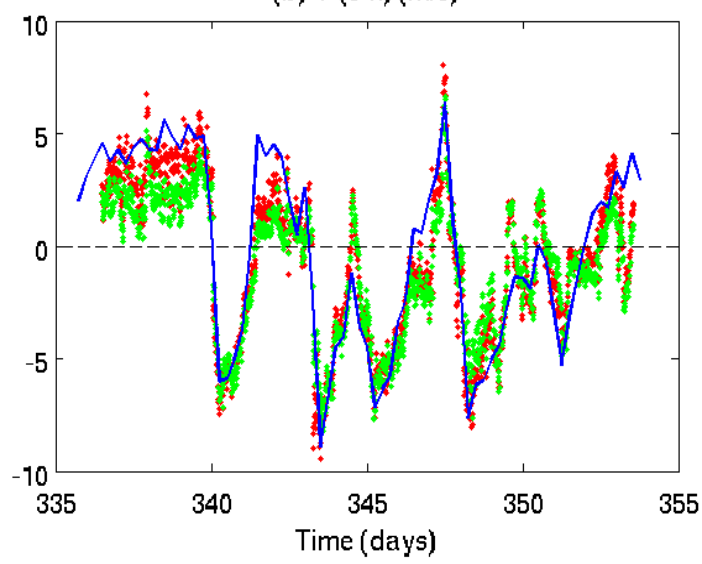

(d) Mixing ratio $(\mathrm{g} / \mathrm{kg})$

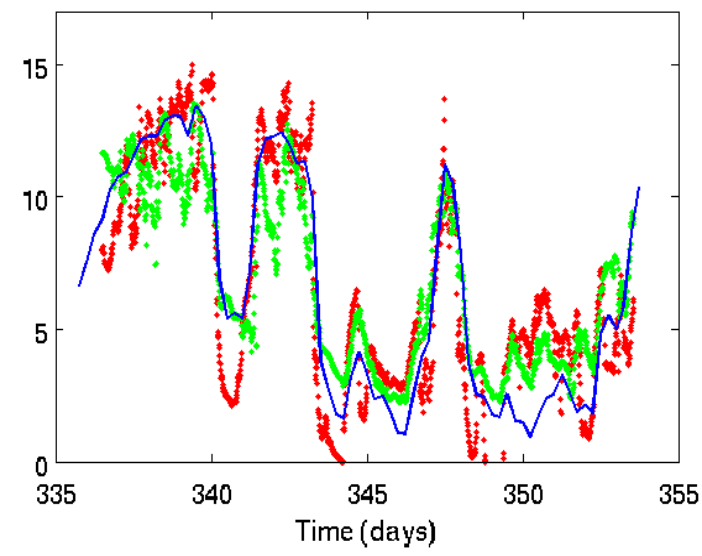

Fig. 4.1: Time series of 15-min mean (a) west-east wind component, (b) south-north wind component, (c) temperature, and (d) water vapor mixing ratio at $10 \mathrm{~m}$ (red dots) and $5 \mathrm{~m}$ (green dots). Blue curve indicates the NCEP FNL analysis data at the grid point closest to the tower site. 
(a) $U_{*}(\mathrm{~m} / \mathrm{s})$

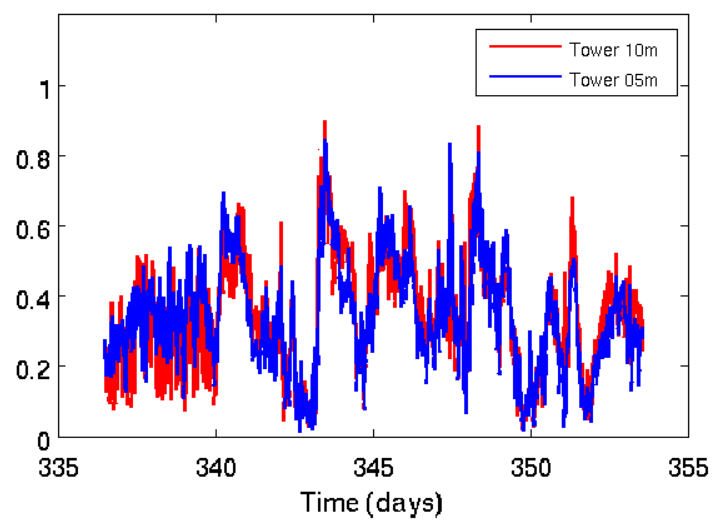

(c) Sensible heat flux $\left(W / \mathrm{m}^{2}\right)$

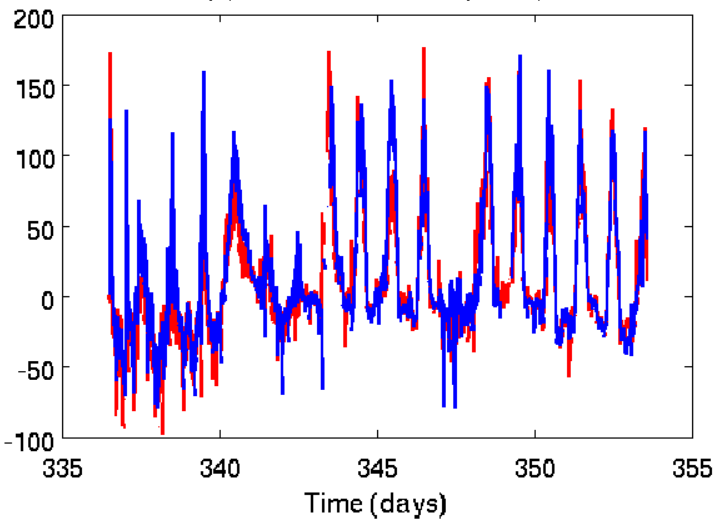

(b) TKE $\left(\mathrm{m}^{2} / \mathrm{s}^{2}\right)$

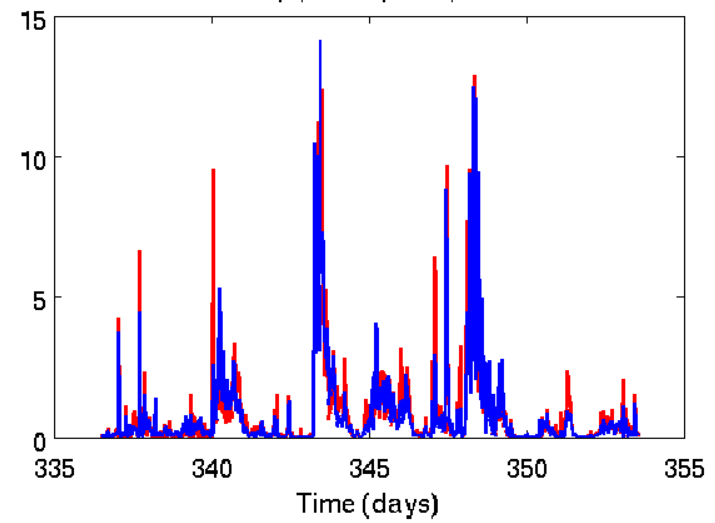

(d) Latent heat flux $\left(W / m^{2}\right)$

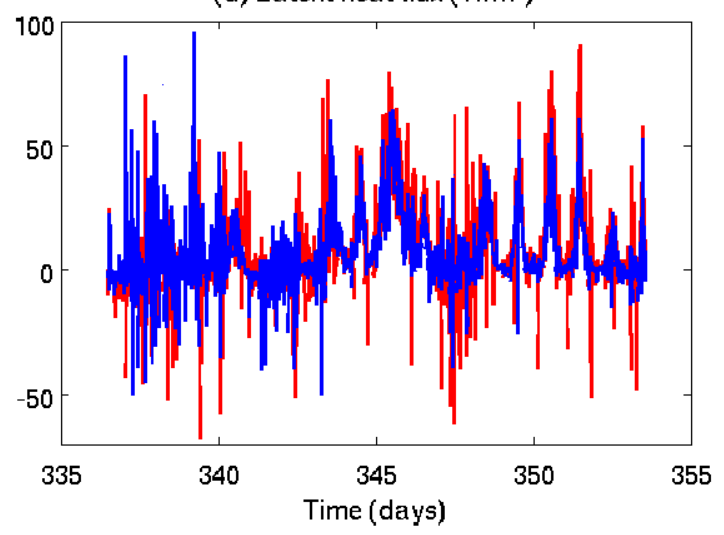

Fig. 4.2: Time series of (a) friction velocity, (b) TKE, (c) sensible heat fluxes, and (d) latent heat fluxes of each 15-min data leg determined by the eddy correlation method at $10 \mathrm{~m}$ (red curve) and $5 \mathrm{~m}$ (blue curve). 
(a) $\mathrm{U}_{*}(\mathrm{~m} / \mathrm{s})$

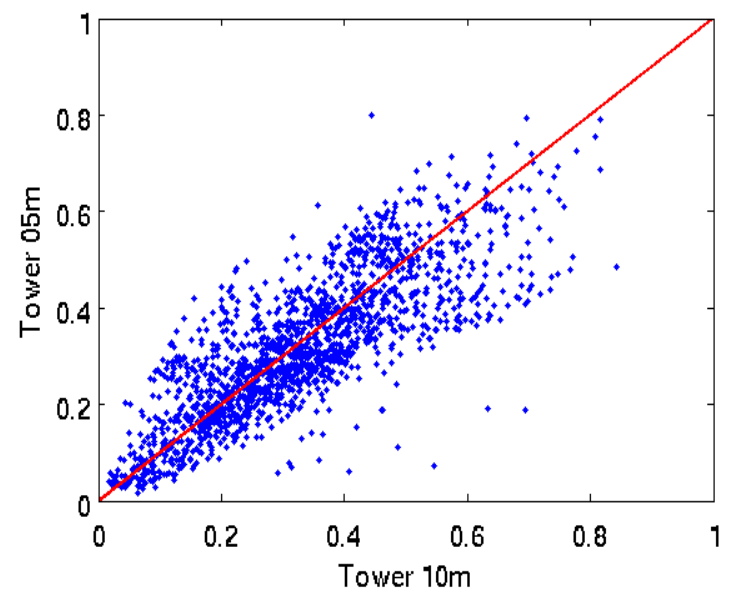

(c) Sensible heat flux ( $\left.W / \mathrm{m}^{2}\right)$

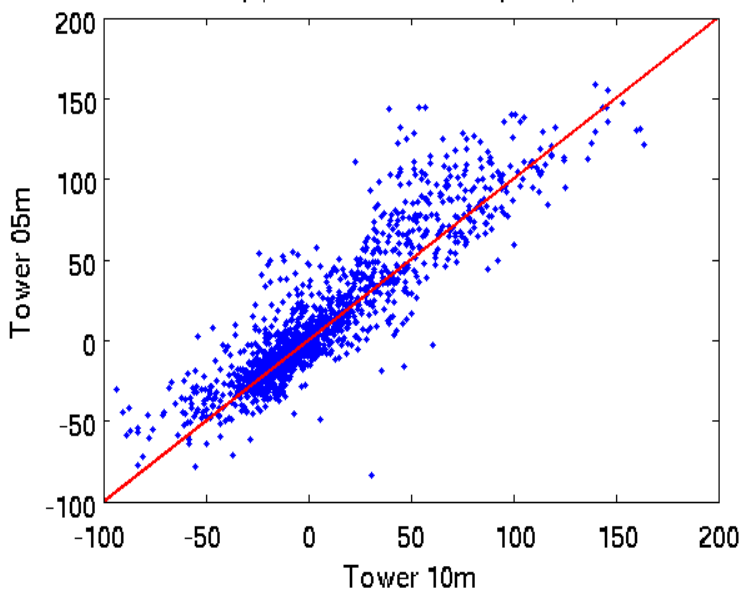

(b) $\operatorname{TKE}\left(\mathrm{m}^{2} / \mathrm{s}^{2}\right)$

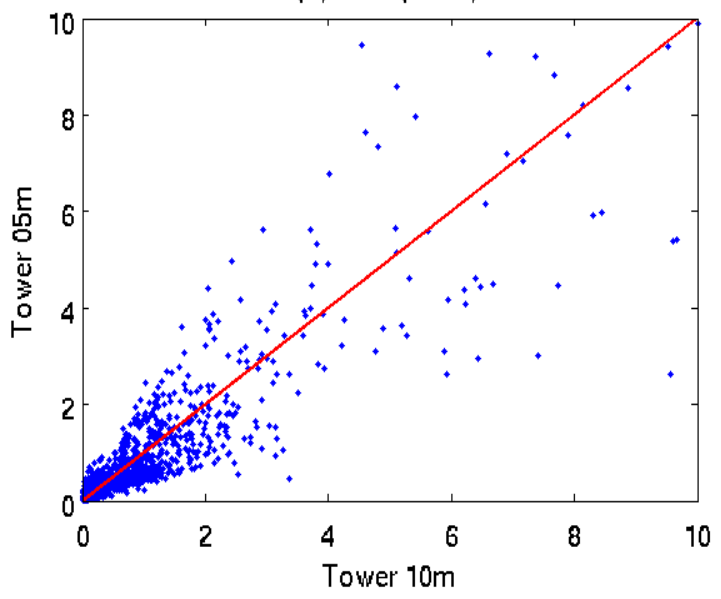

(d) Latent heat flux $\left(W / \mathrm{m}^{2}\right)$

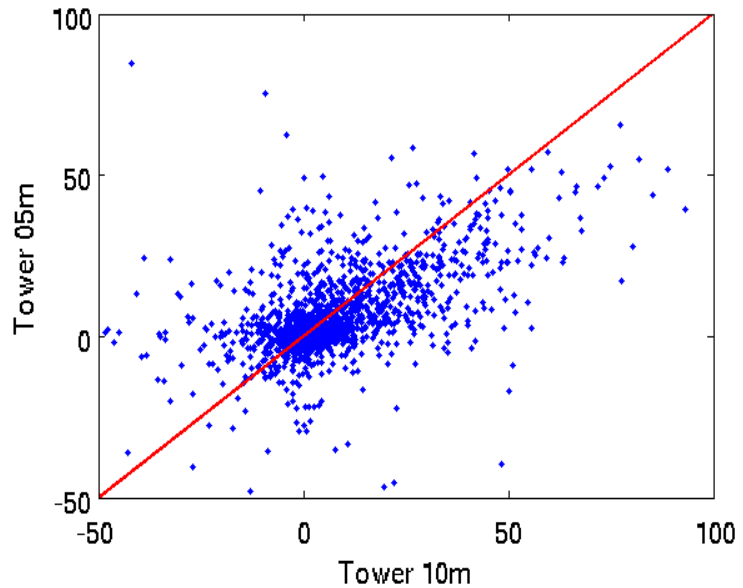

Fig. 4.3: (a) friction velocity, (b) TKE, (c) sensible heat fluxes, and (d) latent heat fluxes of all 15-min data legs at $10 \mathrm{~m}$ against those at $5 \mathrm{~m}$. Red line indicates the constant line. 


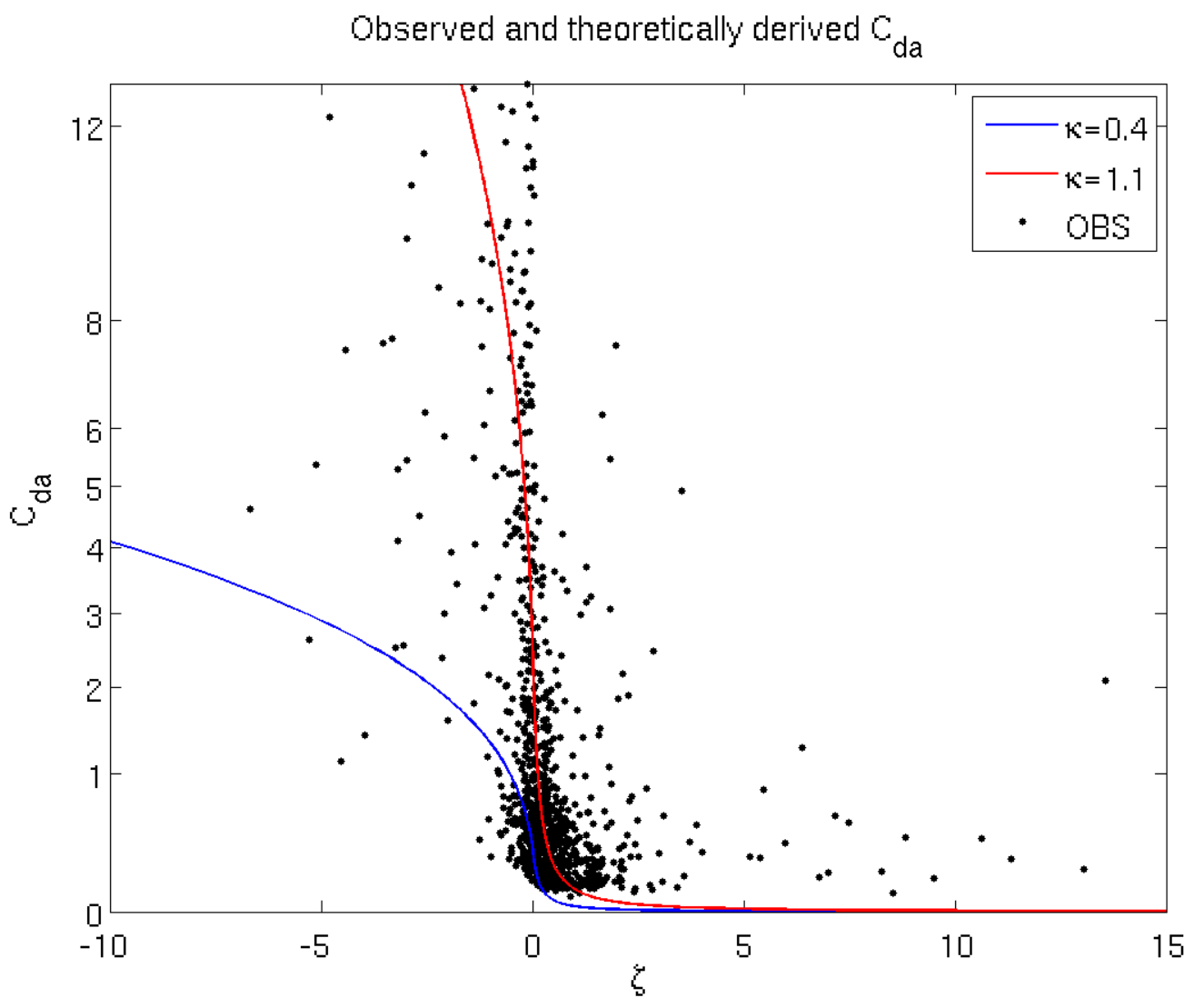

Fig. 4.4: Comparison between observationally determined (dots) and theoretically derived (curves) adjusted drag coefficients. Blue and red curves indicate the theoretical predictions with Von-Karman constant taken as $k=0.4$ and $k=1.1$, respectively. Note that to better illustrate the data, the scale of $C_{D a}$ (y-axis) has been nonlinearly adjusted. 


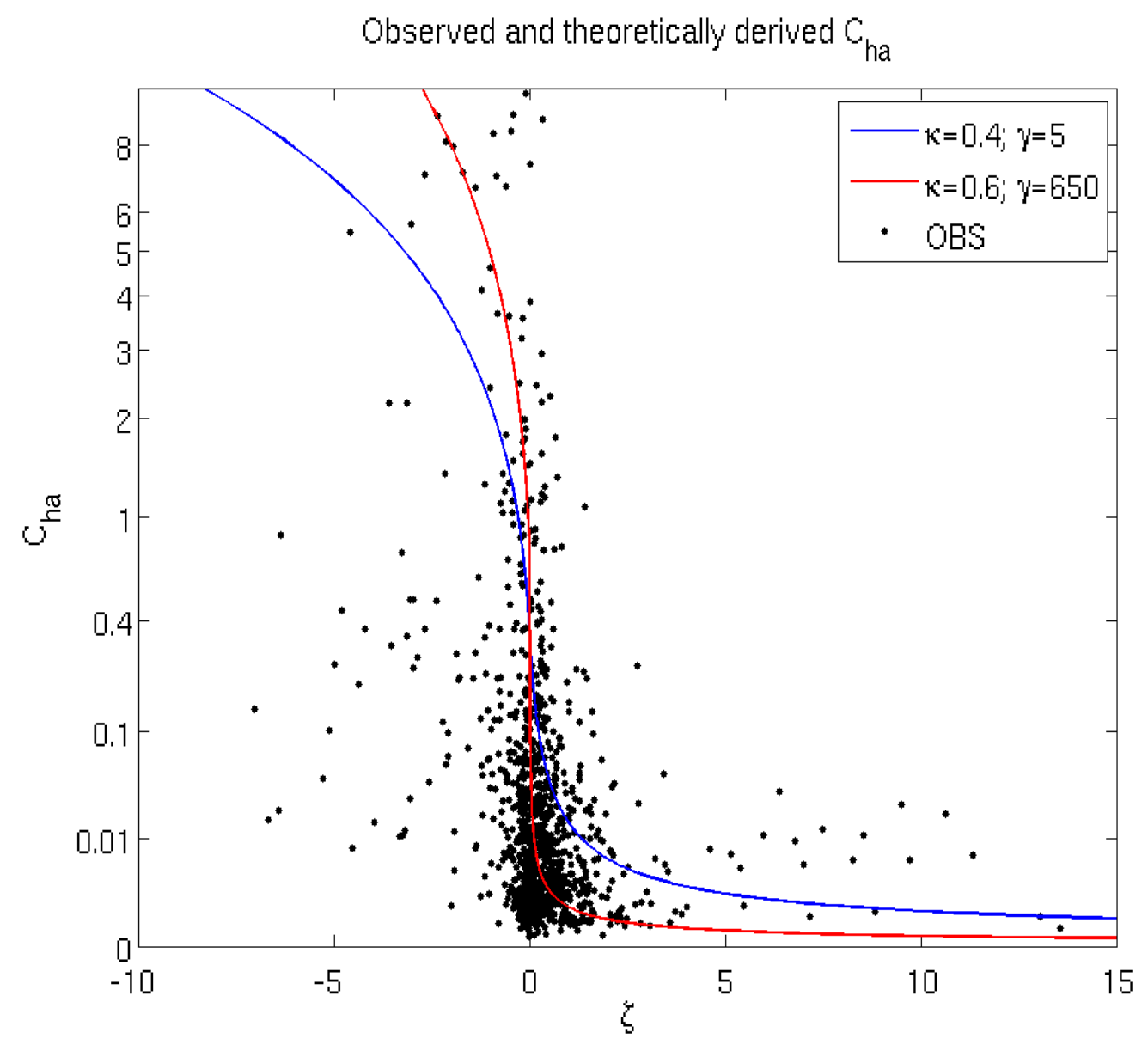

Fig. 4.5: Comparison between observationally determined (dots) and theoretically derived (curves) adjusted exchange coefficient for heat. Blue and red curves indicate the theoretical predictions by the default MOS formula and tuned MOS formula, respectively. Note that to better illustrate the data, the scale of $C_{\mathrm{Ha}}$ (y-axis) has been nonlinearly adjusted. 


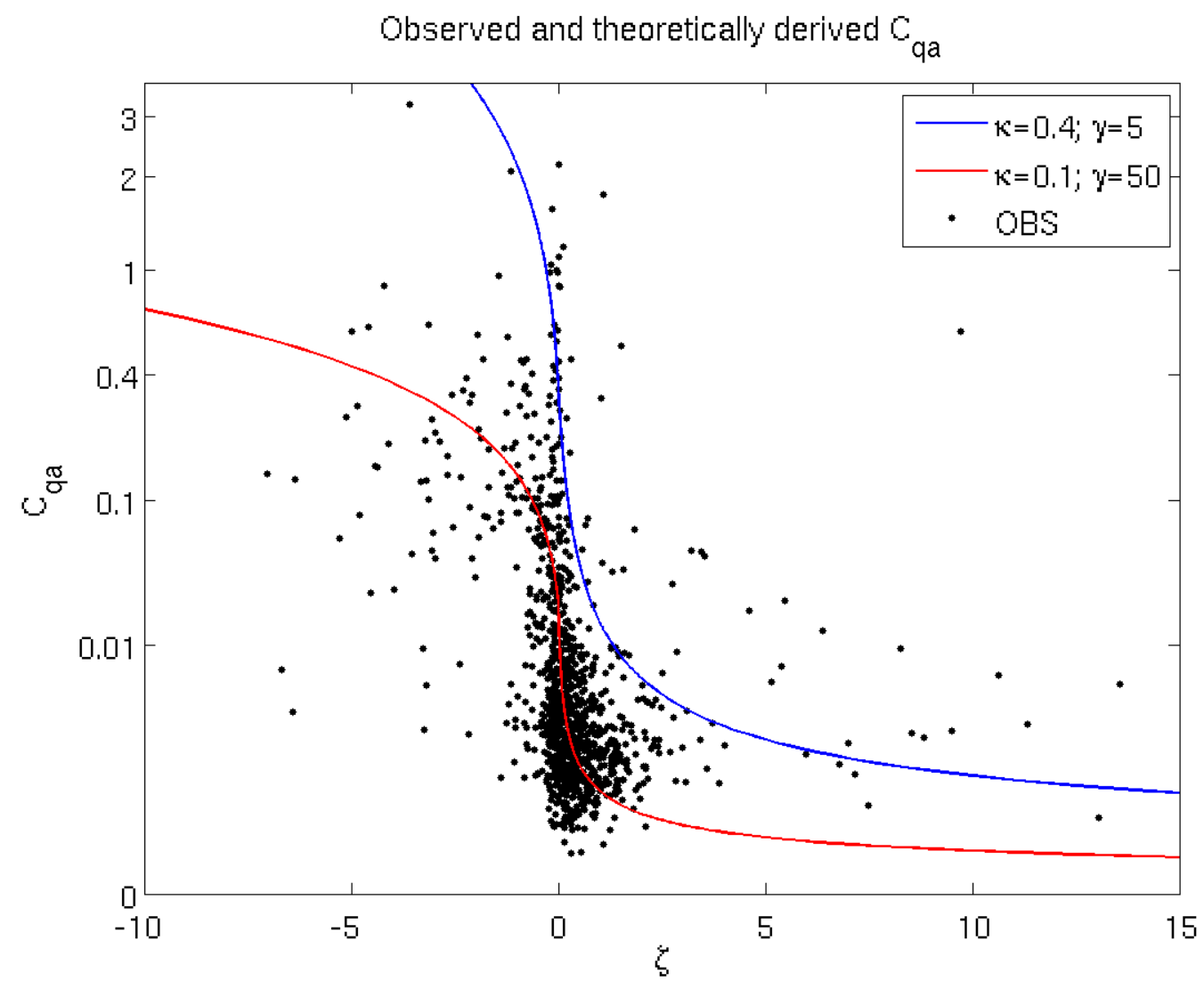

Fig. 4.6: Comparison between observationally determined (dots) and theoretically derived (curves) adjusted exchange coefficient for moisture. Blue and red curves indicate the theoretical predictions by the default MOS formula and tuned MOS formula, respectively. Note that to better illustrate the data, the scale of $C_{Q a}$ (y-axis) has been nonlinearly adjusted. 
(a) $C_{d a}$; wind $<2 \mathrm{~m} / \mathrm{s}$

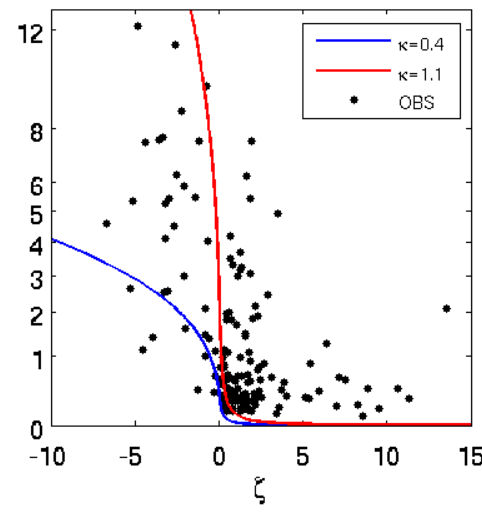

(b) $\mathrm{C}_{\mathrm{da}}$; wind $>2 \mathrm{~m} / \mathrm{s}$

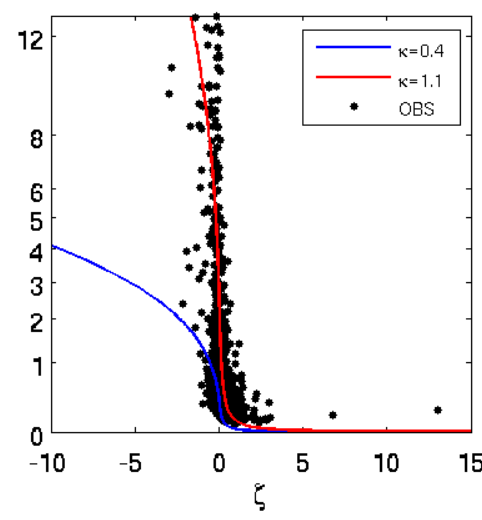

(c) $\mathrm{C}_{\mathrm{ha}}$; wind $<2 \mathrm{~m} / \mathrm{s}$

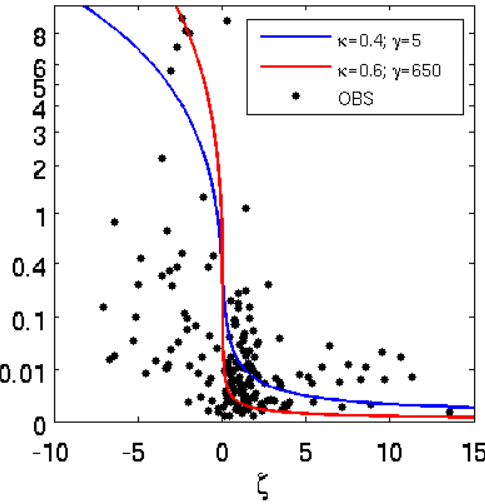

(d) $\mathrm{C}_{\text {ha }}$; wind $>2 \mathrm{~m} / \mathrm{s}$

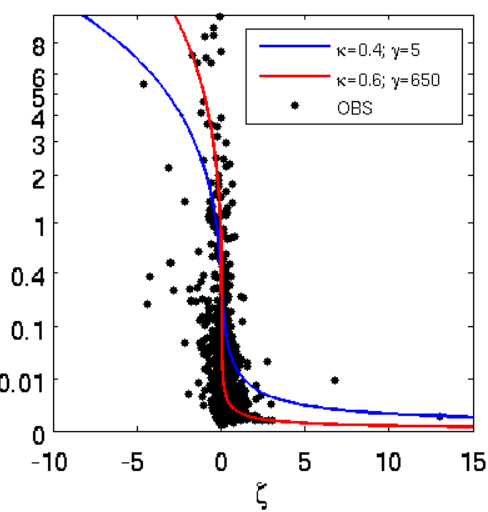

(e) $C_{q a}$; wind $<2 \mathrm{~m} / \mathrm{s}$

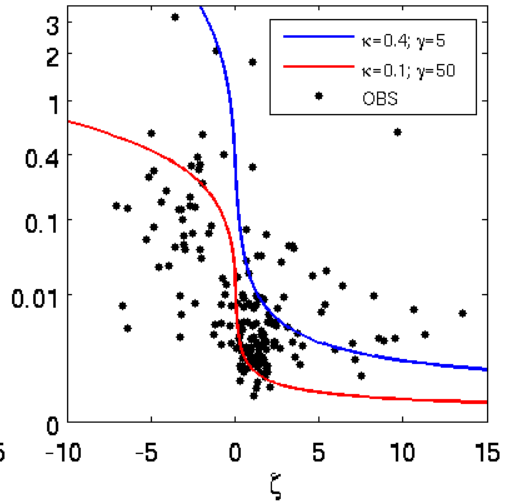

(f) $\mathrm{C}_{\mathrm{qa}}$; wind $>2 \mathrm{~m} / \mathrm{s}$

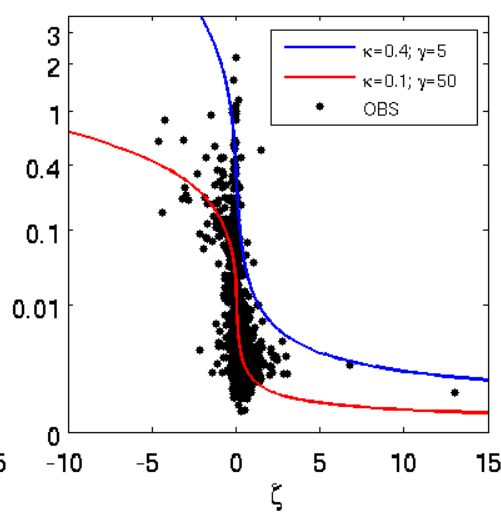

Fig. 4.7: The same data shown in Figs. 4.4-4.6 but grouped into two categories with wind speed smaller and greater than $2 \mathrm{~ms}^{-1}$. 


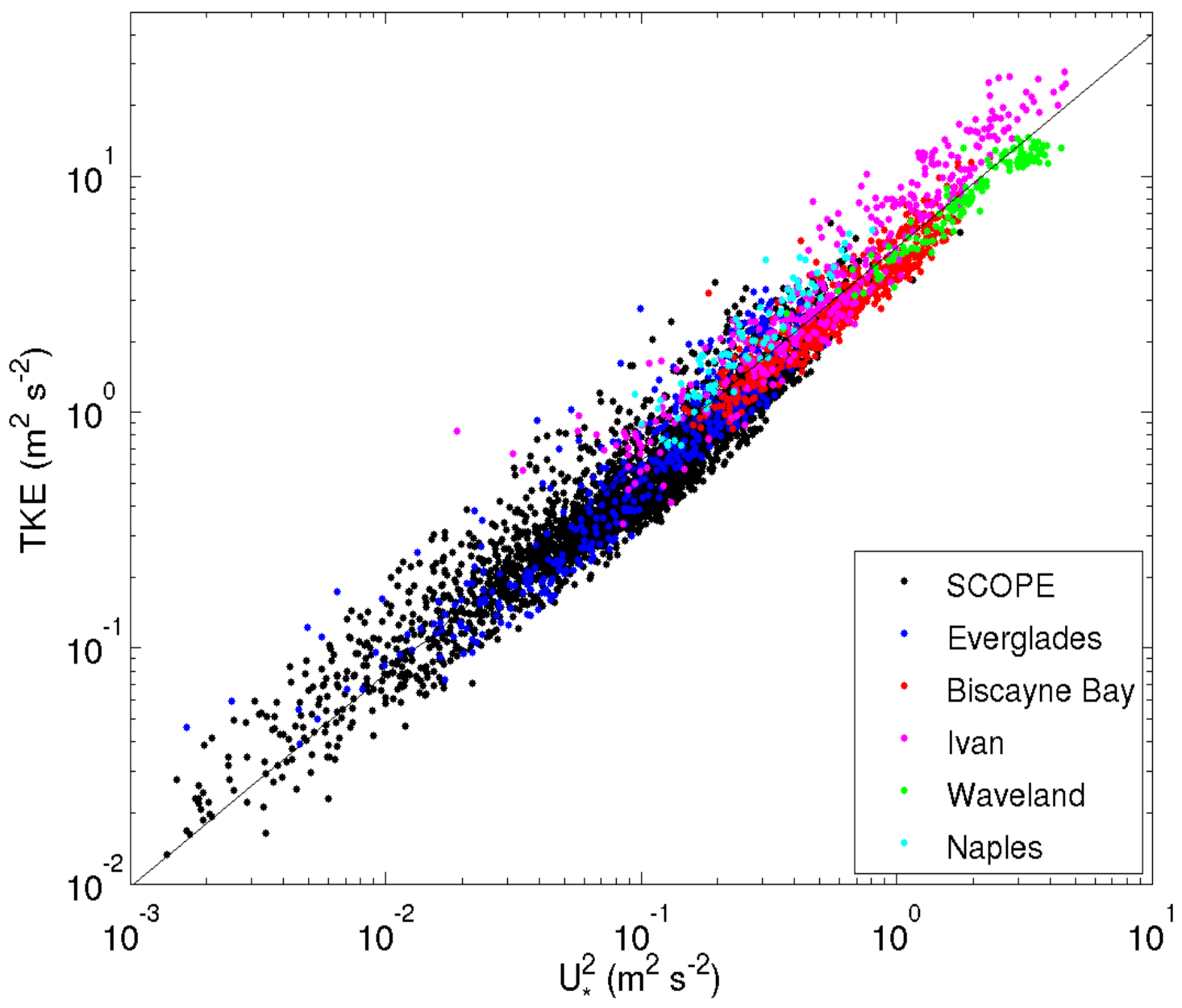

Fig. 4.8: TKE as a function of $u_{*}^{2}$ from different field experiments including SCOPE. The black, blue, red, pink, green cyan dots indicated the data collected during SCOPE and the data collected previously at the Everglades national park, FIU Biscayne Bay campus, during the landfall of Hurricane Ivan, Waveland, and Naples. 


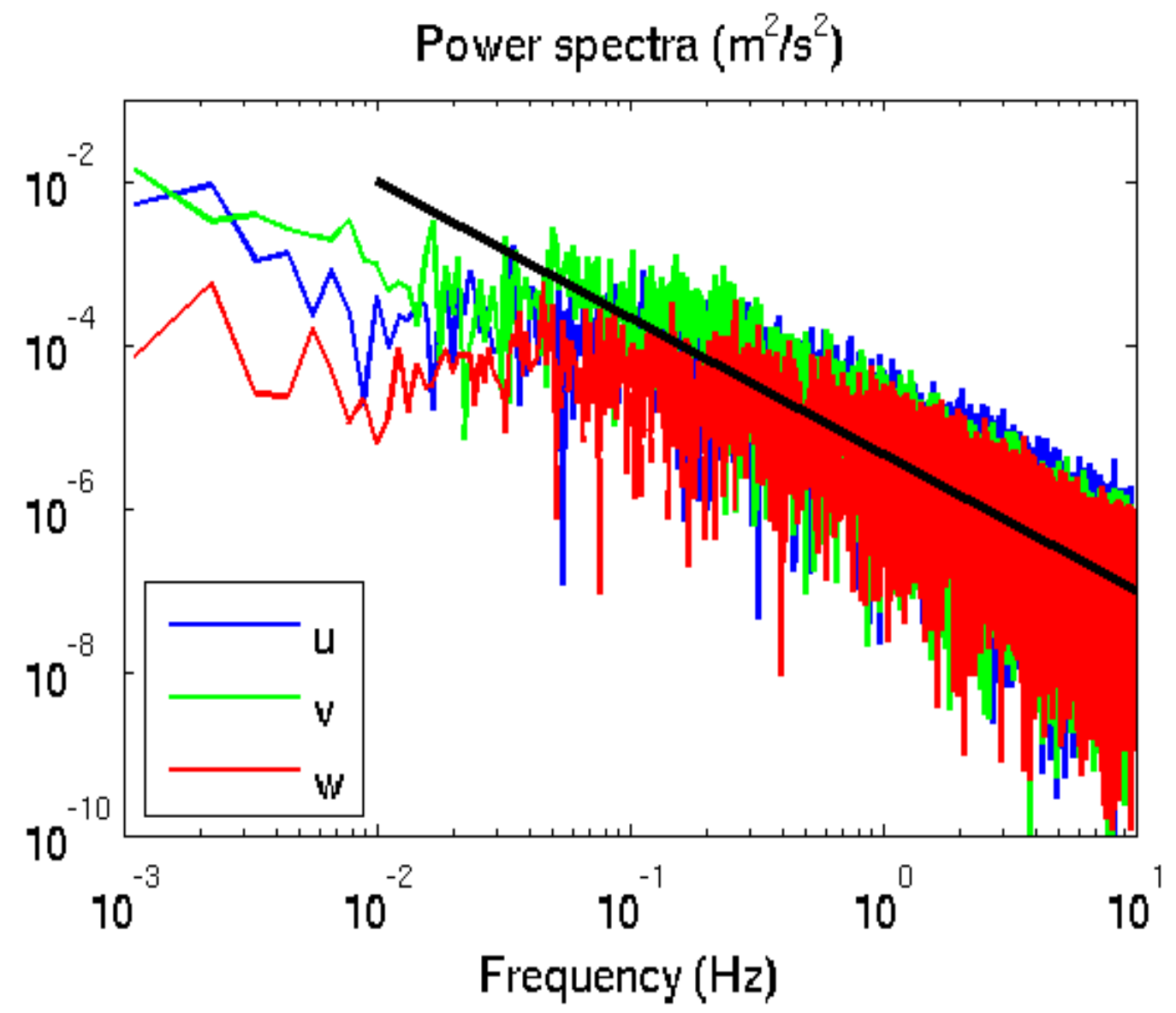

Fig 4.9: Power spectra of $u, v$, and $w$ for an arbitrary 15 minute spectral lag from the SCOPE data. 


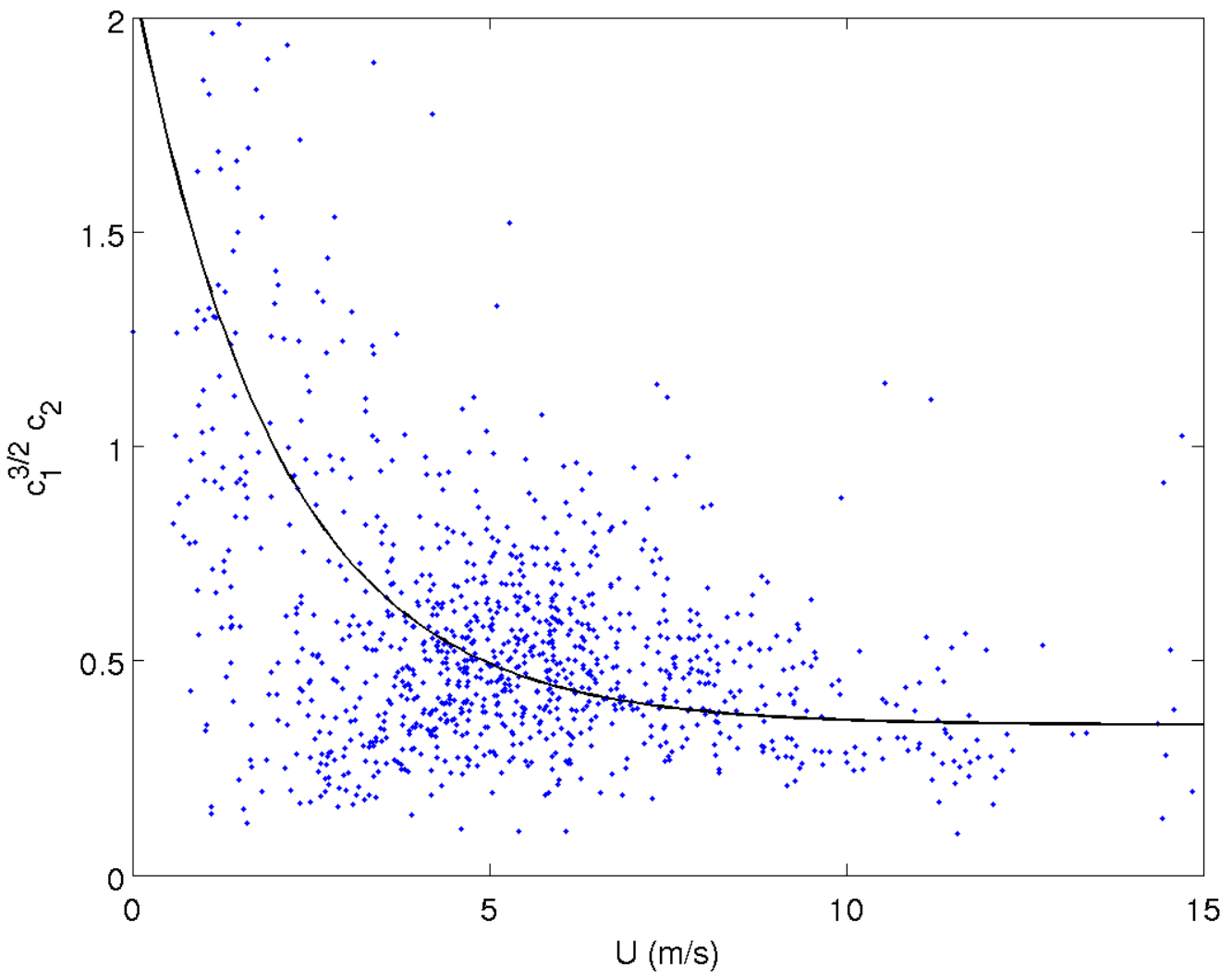

Fig. 4.10: Estimated coefficient $\eta=c_{1}^{3 / 2} c_{2}$ against wind speed for the SCOPE data in near neutral conditions $\left(0.1<\varsigma=\frac{z}{L}<0.1\right)$. 

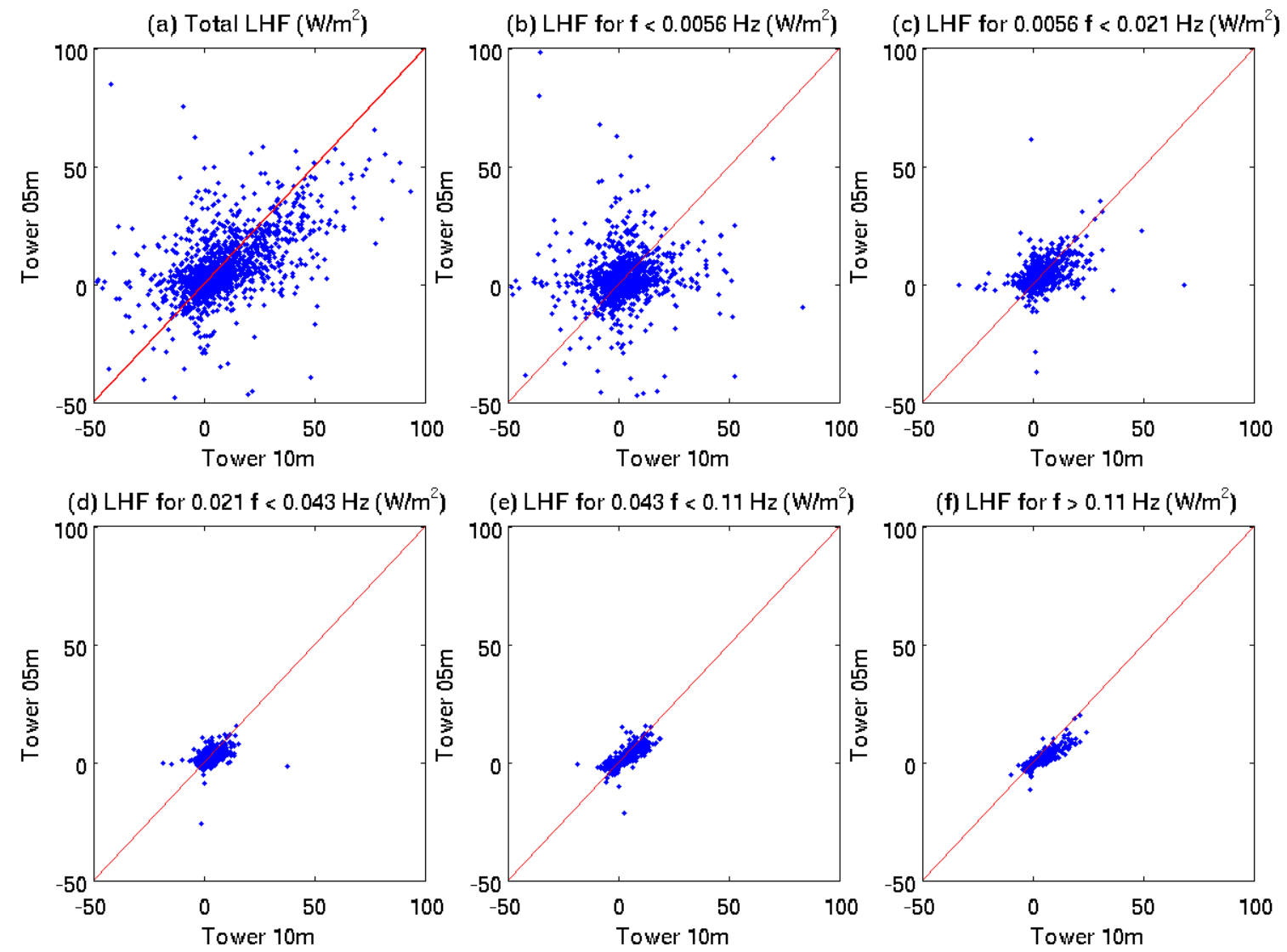

Fig. 4.11: Decomposition of latent heat fluxes in terms of eddy frequencies for all flux legs (15 min) of the SCOPE data. 


\section{Chapter V: SUMMARY AND DISCUSSION}

Vertical turbulent transport in the SL plays a key role in determining the exchange of momentum, heat, and moisture between the atmosphere and the underlying surface. However, in numerical weather forecasting, climate simulation and projection, and many other applications in geosciences, the surface turbulent transport is a sub-grid scale process that can only be determined parametrically. A common method to parameterize SL turbulent fluxes is the bulk transfer model in which the turbulent fluxes are represented in terms of surface mean meteorological variables. While the method itself is simple and provides a practical way to calculate surface fluxes, the exchange coefficients, which relate the turbulent fluxes to mean meteorological variables, cannot be determined by the bulk model itself. In practice, to close the parameterization system, the bulk transfer model is often combined with the MOS theory from which the analytical expressions for exchange coefficients can be derived. Although widely used in numerical simulations and other applications in geosciences, some characteristics of the theoretically derived exchange coefficients from MOS are not supported by observations. Examples of this kind of behavior include the failure of MOS in predicating drag coefficients in overland calm wind conditions (Zhu and Furst 2013). Moreover, the empirical stability functions used in MOS were obtained almost 50 years ago based on the Kansas field experiment taken place on homogeneous wheat fields. It remains to be a question as to if the empirical stability functions obtained from the Kansas field experiment are applicable in different weather and surface conditions other than homogeneous wheat fields in fair-weather conditions. Despite the scientific importance to address the unanswered questions associated with the flux parameterization developed 
from MOS and bulk transfer model, how to provide an appropriate evaluation of the parameterization system using observations poses a great challenge for boundary layer meteorologists. The problem stems from the difficulties not only in obtaining direct turbulent flux data itself but also in measuring surface skin temperature and moisture and surface aerodynamic and thermal roughness, which are needed to calculate turbulent fluxes parametrically from mean variables and theoretically from MOS. Over a complex terrain, surface roughness can depend strongly on wind direction due to surface inhomogeneity. The sharp vertical gradient of temperature and moisture close to the surface, surface radiation, dew, and other factors result in large uncertainties in skin temperature and moisture measurements. Thus, it is practically impossible to provide a meaningful evaluation of surface flux parameterization directly from observations.

To solve the problem in evaluating surface flux parameterization using observations, in this study, a novel method has been developed. This method utilizes the observations collected by the fast-responding sensors at two levels in the SL to examine the formalism of MOS and bulk transfer model. The advantage of this method is that the uncertainties associated with surface aerodynamic and thermal roughness and surface skin temperature and moisture, which can substantially affect the accuracy of calculating exchange coefficients, are excluded, and thus, it can provide an objective evaluation of surface flux parameterization. This method can be readily applied to any surface and weather conditions as long as fast-responding observations are available at two heights in the SL.

The data used in this study was collected during the SCOPE, a nearshore field experiment supported by GoMRI. The IHRC PWT deployed at the Beasley Park, Fort 
Walton Beach, Florida collected the meteorological variable using fast-responding sensors at $5 \mathrm{~m}$ and $10 \mathrm{~m}$ during the entire experimental period from December $3^{\text {rd }}$ to December $20^{\text {th }} 2013$. The goal of this thesis study is provide a comprehensive evaluation of surface flux parameterization using the in-situ tower observations of SCOPE based on the innovative evaluation method developed in this study. The main results are summarized as follows.

1. The theoretical prediction of adjusted exchange coefficients based on the MOS formalism obtained from the well-known Kansas field experiment does not match well with those directly computed from the SCOPE data. However, we showed that the surface flux parameterization can be improved within the existing MOS formalism if some of the assumption made by MOS can be relaxed. For example, tuning Von-Karman constant yields a better fit of the observational data by the theoretical prediction. The physical base for such tuning of parameters used in MOS has been discussed from the TKE budget perspective. We showed that the classic log-wind profile in the SL obtained from the dimensional analysis of MOS can be derived from the TKE budget with appropriate assumptions but ending up with a varying parameter equivalent to VonKarman constant. The argument from the TKE budget perspective is shown to be consistent with the SCOPE data.

2. The large spread in observed adjusted exchange coefficients mainly occurs in the calm wind regime with strong stability. We argued that the fluxes induced by the convective plumes in strong unstable conditions and intermittent turbulence in strong stable conditions are the main culprits that deviate the observations the theoretical predictions by MOS. Previous studies also show that both of the scenarios appeared to be 
problematic for MOS. Thus, attention needs to be paid to the calm-wind conditions in future research.

3. Large turbulent eddies, which depend primarily on the mean flow pattern and surface conditions, tend to break down the constant flux assumption in the SL. We speculate that the constant flux assumption will be substantially affected by the complex terrain and mesoscale and synoptic weather systems and may not be as reliable as that in fair weather condition over the homogeneous terrain. The unique characteristics of large eddies poses a great challenge for including their contribution surface flux.

In this study, although we successfully derived the formulism for momentum transport from the TKE budget equation, how to develop a similar method to account for heat and moisture transport has not been discussed. To explore this possibility will be a focus of my future research. 


\section{REFERENCES}

Aubient, M., T. Vesala, and D. Papale, eds. 2012. Eddy Covariance: A Practical Guide to Measurement and Data Analysis. Springer Atmospheric Sciences.

Black, PG, D'Asaro E, Drennan WM, French JR, Niiler PP, Sanford TB, Terrill EJ, Walsh EJ, Zhang JA (2007) Air-sea exchange in hurricanes: Synthesis of observations from the Coupled Boundary Layer Air-Sea Transfer experiment. Bull Amer Meteorol Soc 88:357-374

Busch, N. E.: 1973. 'The Surface Boundary Layer' (Part I), Boundary-Layer Mereorol. 4,213-240.

Businger, J. A., Wyngaard, J. C., Izumi, I., and Bradley, E. F.: 1971, 'Flux-Profile Relationships in the Atmospheric Surface Layer', J. Atmospheric Sci. 28, 181189.

Christen, A., E. Gorsel, and R. Vogt, (2007): Coherent structures in urban roughness sublayer turbulence. RMS International Journal of Climatology, 27, 1955-1968.

Deacon, E. L.: 1968, 'The Leveling Error in Reynolds Stress Measurement', Bull. Amer. Meteorol. Sot. 49, 836

Deardorff, J. W., 1973: Three-dimendional numerical modeling of the planetary boundary layer. Workshop on Micrometeorology, Amer. Meteor. Soc., Edited by D. Haugen. 271-311.

Donelan M. A., Haus BK, Reul N, Plant WJ, Stianssnie M, Graber HC, Brown OB, Saltzman ES, (2004) On the limiting aerodynamic roughness of the ocean in very strong winds. Geophys Res Lett 31: L18306

A. V. Babanin, I. R. Young, M. L. Banner, and C. McCormick (2005), Wave follower field measurements of the wind input spectral function: Part I. Measurements and calibrations, J. Atmos. Oceanic Technol., 22, 799-813, doi:10.1175/JTECH1725.1.

Dyer, A. J. and Hicks, B. B.: 1972, 'The Spatial Variability of Eddy Fluxes in the Constant Flux Layer', Quart. J. Roy. Meteorol. Sot. 98, 206-212.

Garratt, J. A. (1977): Review of drag coefficients over oceans and continents, Mon. Weather Rev., 105, 915-929.

Grachev, A. A. and co-authors, 2011: Turbulent fluxes and transfer of trace gases from ship-based measurements during TexAQS 2006. J. Geophy. Res., er, D13110. 
Kaimal, J. C., and D. A. Haugen, (1969): Some Errors in the Measurement of Reynolds Stress. J. Appl. Meteor., 8, 825-827

—-, Wyngaard, J. C., Izumi, Y., and Cote, 0. R.: 1972, 'Spectral Characteristics of Surface Layer Turbulence', Quart. J. R. Meteorol. Sot. 98, 563-589.

—, Wyngaard, J. C., Haugen, D. A., Cote, 0. R., Izumi, Y., Caughey, S. J., and Readings, C. J.: 1976, 'Turbulence Structure in the Convective Boundary Layer', J. Atmos. Sci. 33, 2152-2169.

Lanzinger, E., and H. Langmack. Measuring air temperature by using an ultrasonic anemometer. Poster presented at TECO-2005, Bucharest, Romania.

LI-COR Biosciences. (2010). LI-7200 $\quad \mathrm{CO}_{2} / \mathrm{H}_{2} \mathrm{O}$ Analyzer Instruction Manual. Publication No. 984-10564. LI-COR, Inc, Lincoln, NE.

Mahrt L, Vickers D, Sun J, Jensen NO, Jørgensen H, Pardyjak E, Fernando H (2001) Determination of the surface drag coefficient. Boundary-Layer Meteorol 99: 249276

— 2010: Computing Turbulent Fluxes Near the Surface: Needed Improvements. Agr. For. Met. 150, 501-509.

Mellor, G. L. and T. Yamada, (1974): A hierarchy of turbulent closure models for planetary boundary layers. J. Atmos. Sci., 31, 1791-1806.

Monin, A. S., and A. M. Obukhov (1954), Basic laws of turbulent mixing in the ground layer of the atmosphere, Akad. Nauk. SSR Geohiz. Inst. Tr., 151, 1963-1987.

Montgomery, R. B., (1948): Vertical Eddy Flux of Heat in the Atmosphere, J. Meteorol. 5, 265-274.

Pond, S.: 1968, 'Some Effects of Buoy Motion on Measurements of Wind Speed and Stress', J. Geophys. Res. 73, 507-512.

Ridder, K. D. (2010), Bulk Transfer Relations for the Roughness Sublayer, Boun. Lay. Met. 134, 257-267.

Schmid H. P., Grimmond C. S. B., Cropley F., Offerle B., Su H. B., (2000): Measurements of $\mathrm{CO} 2$ and energy fluxes over a mixed hardwood forest in the mid-western United States. Agric For Meteorol., 103, 357-374.

Stull, R. B. (1988), An Introduction to Boundary Layer Meteorology, 666 pp., Kluwer Academic Publishers, Dordrecht. 
Tanner, C. B. and Thurtell, G. W. : 1969, 'Anemoclinometer Measurements of Reynolds Stress and Heat Transport in the Atmospheric Surface Layer', U.S. Army Electronics Command, R and D Tech. Report, ECOM 66-G22-F, 199 pp.

Wang, J., and R. Bras (2009), An Extremum Solution of the Monin-Obukhov Similarity Equations, J. Atmospheric Sci. 67, 485-499.

Wilczak, J. M., S. P. Oncley, S. A. Stage, (2001): Sonic Anemometer Tilt Correction Algorithms. Boundary-Layer Meteorol, 99, 127-150.

Zeman, O. (1981): Progress in the modeling of planetary boundary layers. Annual Rev. in Fluid Mech. 13, 253-272.

Zhu, P., and J. Furst (2013), On the parameterization of surface momentum transport via drag coefficient in low-wind conditions, Geophys. Rex. Lett., 40, 2824-2828. 
Appendix A

IHRC Portable Wind Tower

Instrument Specifications

Table A.1 Instrument specifications for the Gill WindMaster Pro Ultrasonic Anemometer.

Wind Speed

Range: $\quad 0$ to $65 \mathrm{~m} / \mathrm{s}$ (0 to $145 \mathrm{mph}$ )

Resolution: $\quad 0.01$ or $0.001 \mathrm{~m} / \mathrm{s}$

Accuracy $(12 \mathrm{~m} / \mathrm{s}): \quad<1.5 \%$ RMS

Wind Direction

Range:

Resolution:

Accuracy $(12 \mathrm{~m} / \mathrm{s})$ :

Speed of Sound

Range:

Resolution:

Accuracy:

General

Output sample rate:

Sonic temp. range:
0.0 to 359.9 degrees

1 degrees or 0.1 degrees

2 degrees

300 to $370 \mathrm{~m} / \mathrm{s}$

$0.01 \mathrm{~m} / \mathrm{s}$

$< \pm 0.5 \%$ @20 degrees C

up to $32 \mathrm{~Hz}$

-40 to +70 degrees $\mathrm{C}$ 
Table A.2 Instrument specifications for the LiCor LI- $7200 \mathrm{CO}_{2} / \mathrm{H}_{2} \mathrm{O}$ Gas Analyzer System

General

Accuracy: within $2 \%$ of reading

Resolution $\left(\mathrm{H}_{2} \mathrm{O}, \mathrm{CO}_{2}\right)$ : $\quad 0.0067 \mathrm{ppt}$

Operating temp. range: $\quad-25$ to 50 degrees $\mathrm{C}$

Power requirements: $\quad 10.5$ to 30 volts DC

Power consumption: $\quad 12 \mathrm{~W}$ nominal

Path length: $\quad 12.5 \mathrm{~cm}$

Bandwidth: $\quad$ up to $20 \mathrm{~Hz}$

RH range: $\quad 0-95 \%$ (non-condensing) 